\title{
ASPECTS MORPHOLOGIQUE ET TECHNIQUE DES BOUCLIERS MUSULMANS ${ }^{1}$
}

\author{
PAR \\ LUDVIK KALUS
}

DANS le numéro précédent de cette revue, nous avons présenté l'évolution des boucliers circulaires de l'Orient musulman ${ }^{2}$, en essayant de suivre d'une façon générale le développement des éléments caractéristiques de ces boucliers à travers diverses époques et dans divers milieux culturels et géographiques et en s'efforçant de définir des groupes de boucliers typiques pour une telle époque ou pour un tel milieu. Cette étude était basée sur une analyse approfondie d'une centaine de boucliers que nous avons eu l'occasion d'étudier dans plusieurs musées d'Europe ${ }^{3}$, ainsi que sur des ouvrages et articles dans lesquels se trouvent des renseignements concernant les boucliers circulaires musulmans ${ }^{4}$.

Les travaux préliminaires pour cette étude nous ont amené à observer d'une façon détaillée la forme et la structure de chacun des boucliers étudiés, c'est-à-dire l'apparence extérieure de tous les éléments les composant ainsi que l'arrangement de tous ces éléments ensemble. Ces éléments, nous les appelons éléments morphologiques. En examinant et en comparant tous les éléments morphologiques des boucliers entre eux, nous procédons dans la présente étude à l'analyse morphologique des boucliers circulaires de l'Orient musulman en tant qu'espèce, dans le cadre de la "population» examinée, sans tenir compte de leur évolution

1 Cet article constitue le premier chapitre de la deuxième partie de notre thèse de doctorat de $3^{\mathrm{E}}$ cycle, dirigée par Mme Janine Sourdel-Thomine, professeur d'art et archéologie islamiques à Paris-Sorbonne et directeur d'études d'épigraphie et paléographie arabes à l'Ephe (IV section), thèse présentée en 1974 à l'Université de Paris-Sorbonne (Paris IV), sous le titre Contribution à l'étude des boucliers circulaires de l'Orient musulman.

${ }^{2}$ Ludvik Kalus: Boucliers circulaires de l'Orient musulman (évolution et utilisation), dans Gladius, XII, 1974, pp. 59-133, 30 fig.

${ }^{3}$ Les musées visités sont les suivants: Historisches Museum de Berne (Suisse), Musće de l'Armée de Paris, Musée de l'Homme de Paris (France), Kunsthistorisches Museum de Vienne, Historisches Museum der Stadt Wien, Heeresgeschichtliches Museum de Vienne (Autriche), Naprstkovo Museum de Prague (Tchécoslovaquie). Nous tenons à exprimer encore une fois nos remerciements aux directions respectives de ces musées d'avoir eu la gentillesse de nous autoriser à étudier «de près» les objets faisant partie de leurs collections.

${ }^{4}$ Pour la bibliographie de ces ouvrages et articles voir Ludvik Kadus: Boucliers circulaires de l'Orient musulman (évolution et utilisation), dans GLADIUS, XII, 1974 , pp. 127-133. 
historique. Il s'agit en principe de l'énumération et de la classification des divers éléments morphologiques, accompagnées de l'observation de leurs variétés et de leurs types. Une telle approche nous permet de suivre de près le côté technique des boucliers et nous permet d'observer de plus près la modification de certains éléments des boucliers qui allait de paire avec le changement de l'utilisation des boucliers ${ }^{5}$.

\section{GÉNÉRALITÉS SUR LA COMPOSITION}

D'UN BOUCLIER CIRCULAIRE

Un bouclier est composé, en général, d'une base et de parties rapportées sur cette base.

La base du bouclier est la plaque ronde qui sert à protéger le corps du combattant ou une de ses parties. Le côté de la base qui, si le bouclier est porté, est tourné vers l'extérieur, est appelé surface externe (ce côté est presque toujours convexe) et son autre côté, tourné vers le combattant, surface interne. La surface que présente l'épaisseur de la base du bouclier sur tout son pourtour sera appelée tranche ${ }^{6}$.

Les surfaces externe et interne du bouclier circulaire sont divisées par le profilage et souvent aussi par l'arrangement du décor en trois parties principales ${ }^{7}$ (Fig. 1). Le centre du bouclier, autour du sommet de la convexité, est appelé umbo (ou ombilic), il est parfois pourvu d'une pointe saillante qui fait partie intégrale de la base ou bien est rapportée sur elle. L'umbo peut être limité, soit par la structure morphologique de la base (voir plus loin les boucliers en osier tressé), soit par le cadre décoratif d'un médaillon central, ou bien ses limites ne sont pas désignées et l'umbo se perd dans les flancs (qui peuvent être aussi appelés champ) du bouclier. Ce terme comprend pour nous la surface située entre l'umbo et le bord externe du bouclier. Ce bord peut être désigné par le profilage de la base ou par une bordure décorative.

${ }^{5}$ Sur les changement de l'utilisation des boucliers voir Ludvik KaLus: Boucliers circulaires de l'Orienl musulman (évolution el uilisation), dans GLADJus, XII, 1974, pp. 119-126, partie intitulée Utilisation des boucliers circulaires de l'Orient musulman.

- Ce terme a été adopté d'après la définition donnée dans le Nouveau Petit Larousse en couleutrs, Paris, 1968, p. 937, article «tranche»:... «Surface que présente un corps vu dans le sens de l'épaisseur».

${ }^{7}$ Cette division ainsi que la terminologie utilisée furent adoptées, après certains changements dûs aux particularités des boucliers d'un autre monde qu'européen, d'après les ouvrages suivants: COMTE DE CHESNEL: Dictionnaire des armées de terre et de mer; encyclopédie militaire et maritime, t. I, Paris, 1862, p. 174, et MaUricF. Albert, Clipeus ou clipeum, article dans Dictionnaire des antiquités grecques et romaines, t. I, 2ème partie, Paris, 1887, pp. 1248-1260. 
Les matériaux utilisés pour la fabrication de la base du bouclier sont très divers, suivant les conditions naturelles et le niveau technique de la société où il était fabriqué, suivant les moyens du propriétaire et les traditions du milieu. Ils changent aussi selon la destination du bouclier lui-même: arme de guerre ou objet à valeur décorative. On a utilisé le plus souvent la fer, l'acier, les peaux de divers animaux, le bois, etc.

Les diamètres des bases circulaires sont très variables, de plus la base est presque toujours profilée. Sa surface externe devient alors convexc

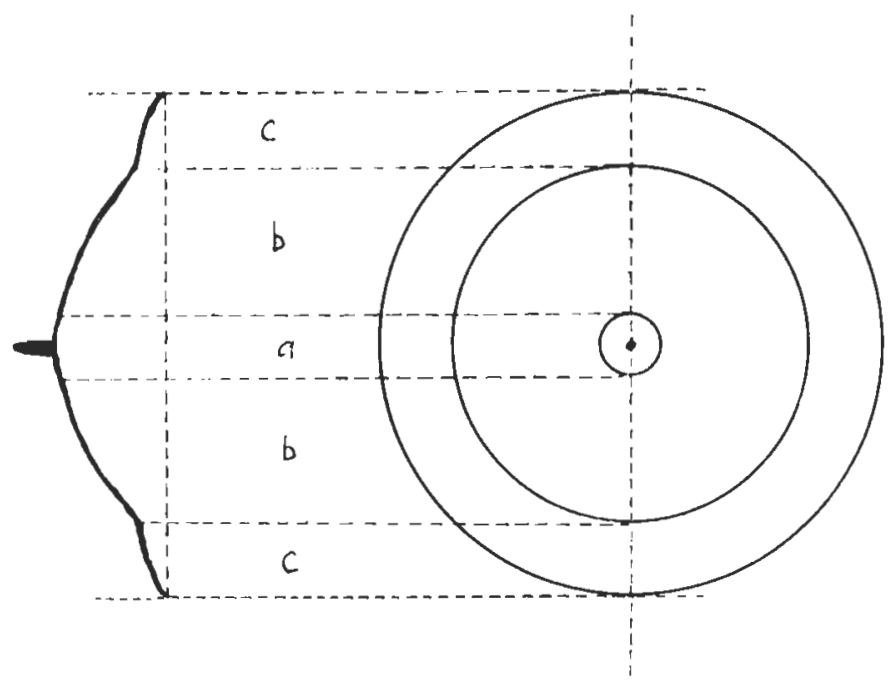

Fic. 1.-Division de la base du bouclier. ( $a=$ umbo pourvu d'une pointe saillante; $b=$ flancs; $c=$ bord.)

et sa surface interne concave. Ce profilage peut donner à la base des formes diverses, par exemple la forme d'une calotte, d'un hémisphère, etc.

La base du bouclier est habituellement pourvue de parties rapportées qui forment avec elle une unité, qui est le bouclier dans toute la force du terme. Elles peuvent être divisées, d'après leur rôle, en groupes suivants: Le premier est le mécanisme qui permet de porter et de manier le bouclier. Il sert à fixer sur la base une lanière ou une poignée, éventuellement une courroie, par laquelle le bouclier est tenu. L'autre groupe des parties rapportées est formé d'éléments renforçant la solidité et l'efficacité du bouclier. Posés et fixés sur la base, ces éléments doivent en 
empêcher la rupture, ainsi qu'il doivent freiner le glissement de l'arme offensive de l'adversaire au-delà du bord du bouclier où elle pourrait menacer le porteur du bouclier. Quant au dernier groupe, il est représenté par les éléments qui doivent amortir les chocs. Ces éléments doivent adoucir le contact entre le bouclier et la main de son porteur ou toute autre partie de son corps en cas de coup porté au bouclier. Pour cela on a surtout recours à unc matelassure qui couvre toute la surface interne de la base ou encore à un coussinet fixé sur l'umbo au-dessous de la poignée.

\section{MÉTHOde ET PRINCIPES D'ANALYSE MORPHOLOGIQUE DES BOUCLIERS ÉTUDIÉS}

Le but de cette analyse est de donner une description et une explication des mécanismes et des éléments formant un bouclier. Nous allons présenter ici leurs différents types en observant ce qu'ils ont de commun et en soulignant les exceptions intéressantes.

Nous nous sommes aperçus, pendant nos travaux préliminaires, que la plupart de ces éléments et mécanismes sont déterminés, dans une certaine mesure, par le matériau utilisé pour la base et varient plus ou moins d'un matériau à l'autre. C'est la raison pour laquelle nous avons basé la division principale des boucliers sur la distinction des matériaux de leur base et c'est dans le cadre des groupes ainsi déterminés que nos observations vont se développer. Il nous a été impossible de faire faire l'analyse chimique des bases des boucliers, ce qui aurait été sans doute très important, surtout pour les boucliers dont la base est en métal ou en peau d'animal. Ainsi la détermination des matériaux utilisés n'a pu être faite que visuellement, ce qui ne nous permet pas une distinction détaillée.

Dans le cadre des groupes déterminés par le matériau de la base, nous allons observer la forme de la base ainsi que les données d'ordre métrologique la concernant (diamètre, profondeur, épaisseur). Quant aux parties rapportées, nous allons observer leurs types, expliquer leur fonctionnement, donner leurs descriptions et, éventuellement, constater leur absence sur certains boucliers.

Les résultats ainsi obtenus pour chaque groupe déterminé par le matériau de la base seront par la suite comparés ensemble et, dans la dernière partie de cet article, nous essayerons de trouver les éléments communs à tout les groupes ainsi que de révéler les éléments distincts et typiques pour chacun d'eux. 
ANALyse MORPHOLOGIQUE DES BOUCliERs EXAMINÉS

\section{Boucliers en métal}

Les principaux métaux utilisés pour la fabrication de la base des boucliers examinés sont: l'acier ordinaire ${ }^{8}$, dont la qualité varie d'un bouclier

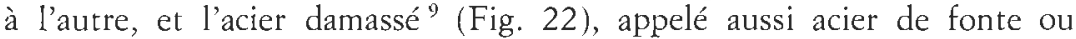
acier de cristallisation ${ }^{10}$. Ce dernier était fabriqué surtout en Iran, à partir de l'acier brut (Wootz) importé de l'Inde. Quand le procédé de la cristallisation était terminé et la forme définitive de l'objet donnée, on faisait apparaître sur la surface de l'acier, par l'action d'un acide, un dessin qui était typique pour un acier damassé mais qui pouvait varier dans les détails d'après le procédé utilisé pendant la cristallisation, tant dans le motif que dans la couleur. Ce dessin permet de distinguer visuellement, à l'aide d'un loupe, l'acier damassé de l'acier ordinaire, ainsi qu'éventuellement l'espèce de l'acier damassé ". Un autre métal utilisé pour la fabrication de la base du bouclier, quoique rarement, est le laiton ${ }^{12}$, qui est un alliage à base de cuivre et de zinc. «La teinte agréable des laitons, variant du rose au jaune selon la teneur en zinc, leur bonne résistance à la corrosion et leur aptitude aux traitements de surface permettent de réaliser économiquement des objets de belle présentation, d'un usage durable et d'un entretien facile» ${ }^{13}$. (Fig. 23.)

"Prague, Musée Naprstek, nos. 19 479, 4 495, 4 505, 4 453, 19 478, 4 506, A 2681, 16 266, 4 504, 4 507, A 23 et 4 680; Berne, Musée d'Histoire, nos. 926, s. n., Pers. 39, 1371, 966 et 1356; Paris, Musée de l'Armée, nos. I 6074, G 739, I. 6670 et I 6999; Paris, Musée de l'Homme, nos. 62.71.29, 47.29.4, 34.141.2 et 43. 27.349; Vienne, Kunsthistorisches Museum, nos. C 191, C 85 et C 94.

"Prague, Musée Naprstek, nos. A 2680 et 43 604; Berne, Musée d'Histoire, nos. 965, 910, 939, 904 et 931; Paris, Musée de l'Armée, no. 2852.

iit Les remarques suivantes concernant l'acier damassé sont basées surtout sur les articles de R. Zeller: Ueber den Damast = Stabl orientaliscber Klingen der Sammlung Henri Moser = Charlottenfels, dans Jabrbucb des Berniscben Historircben Museums, IV, 1924, pp. 110-121; JaKOB von FALKE: Metall-und Schmuckarbeiten des Orients, dans Zeitscbritt für bildende Kunst, lère série, XIII, 1878, pp. 98-100; Jerzy Podosk1: Legenda damastów, dans Broń i Barwa, II, 1935, pp. 269-275. Pour la bibliographie concernant ce problème voir K. A. C. Creswell: A bibliograpby of the archilecture, arls and cratts of Islam to 1st Jan. 1960, Caire, 1961, chapitre «Arms and armour», article «Metallurgy: 'Wootz', or Indian Steel'Damascus' and Persian Sword Blades, etc.», pp. 586-591.

"Au XIX" siècle ce dessin a été imité à Ispahan par l'action de l'acide sans qu'il y ait eu avant le procédé de cristallisation, et l'acier utilisé était d'une qualité infériemre, voir S. G. W. Benjamin: Persia and the Persians, Londres, 1887, pp. 302-303

${ }_{12}$ Berne, Musée d'Histoire, nos. 1370 et s. n.

1. Encyclopaedia Universalis, t. 5, Paris, 1970, article «Cuivre», «3. Alliages de cuivre», «Laitons», p. 220. 
Construction de la base.-La base des boucliers en métal est d'habitude fabri -iuée d'une seule pièce. Néanmoins, certains boucliers sont fabriqués de deux pièces ${ }^{14}$ : la première est formée par un disque qui comprend l'umbo et les flancs, la deuxième est formée par une bande circulaire comprenant le bord. Ces deux pièces sont rivetées et martelées ensemble et la jonction entre elles est d'habitude faite si soigneusement qu'elle ne laisse presque aucune trace sur la surface externe, surtout si elle est recouverte d'un décor dont les limites champ - bord correspondent souvent à la jonction de ces deux pièces.

La base d'un bouclier ${ }^{15}$ est formée de deux pièces jointes qui sont en matériaux différents. Tandis que la partie centrale est en acier damassé, le bord est en acier ordinaire.

Une autre exception est le bouclier ${ }^{16}$ dont l'umbo est pourvu, sur la surface externe, d'une plaque circulaire, concave, terminée au milieu par une pointe saillante, rivetée sur la base du bouclier et accentuant ainsi son umbo.

Dans un seul cas ${ }^{17}$, le bouclier est renforcé sur le bord de sa surface interne par une bande circulaire en fer (sa largeur est de $70 \mathrm{~mm}$ et son épaisseur est de $1,5 \mathrm{~mm}$ ) qui est rivetée sur la base.

Profil de la base.--La base des boucliers peut prendre, dans la plupart des cas par martelage, diverses formes bombées qui sont, approximativement, les suivantes (Fig. 2): la forme d'une calotte sphérique ${ }^{18}$ (Fig. 2a), la forme d'une calotte sphérique légèrement aplatie sur le bord ${ }^{19}$ (Fig. 2b), la forme d'une calotte sphérique dont le bord aplati se détache brusquement de la calotte ${ }^{20}$ (Fig. $2 \mathrm{c}$ ) et la forme d'une calotte sphérique aplatie sur le sommet ainsi que sur le bord ${ }^{21}$ (Fig. 2d). Deux des bou-

It Prague, Musée Naprstek, nos. A 2680, 19 479, 4 505, 43604 et A 2681; Bernc, Muséc d'Histoirc, nos. 939, 904, 931 et s. n.; Paris, Musée de l'Homme, no. 62.71.29.

${ }^{15}$ Berne, Musée d'Histoire, no. 939.

16 Vienne, Kunsthistorisches Museum, no. C 94.

17 Paris, Musée de l'Armée, no. 2852.

${ }^{16}$ Prague, Musée Naprstek, no. 16 266; Berne, Musée d'Histoire, nos. 965, 910, 966 et 1 393; Paris, Musée de l'Armée, nos. I 6074 et I 6999; Vienne, Kunsthistorisches Museum, nos. C 85 et C 94.

${ }^{19}$ Prague, Musée Naprstek, nos. 19 479, 4 495, 4 505, 4 453, A 2681 et 4504 ; Berne, Musée d'Histoire, no. Pers. 39; Paris, Musée de l'Armée, no. I 6670; Paris, Musée de l'Homme, nos. 47.29.4 et 34.141.2.

${ }^{20}$ Prague, Musée Naprstek, nos. A 2680 et 19 478; Berne, Musée d'Histoire, nos. 939, 904 et s. n.; Paris, Musée de l'Homme, no. 62.71.29.

${ }_{21}$ Prague, Musée Naprstek, nos. 4506,43604 et 4 680; Berne, Musée d'Histoire, no. 931; Paris, Musée de l'Armée, nos. 2852 et G 739. 
cliers en forme de calotte sphérique ${ }^{22}$ sont pourvus sur l'umbo d'une pointe saillante qui est, quant au premier, ajoutée et rivetée et, quant au deuxième, la pointe saillante y est une partie intégrale de la base. Deux formes de profil de la base sont exceptionnelles, c'est la forme d'une

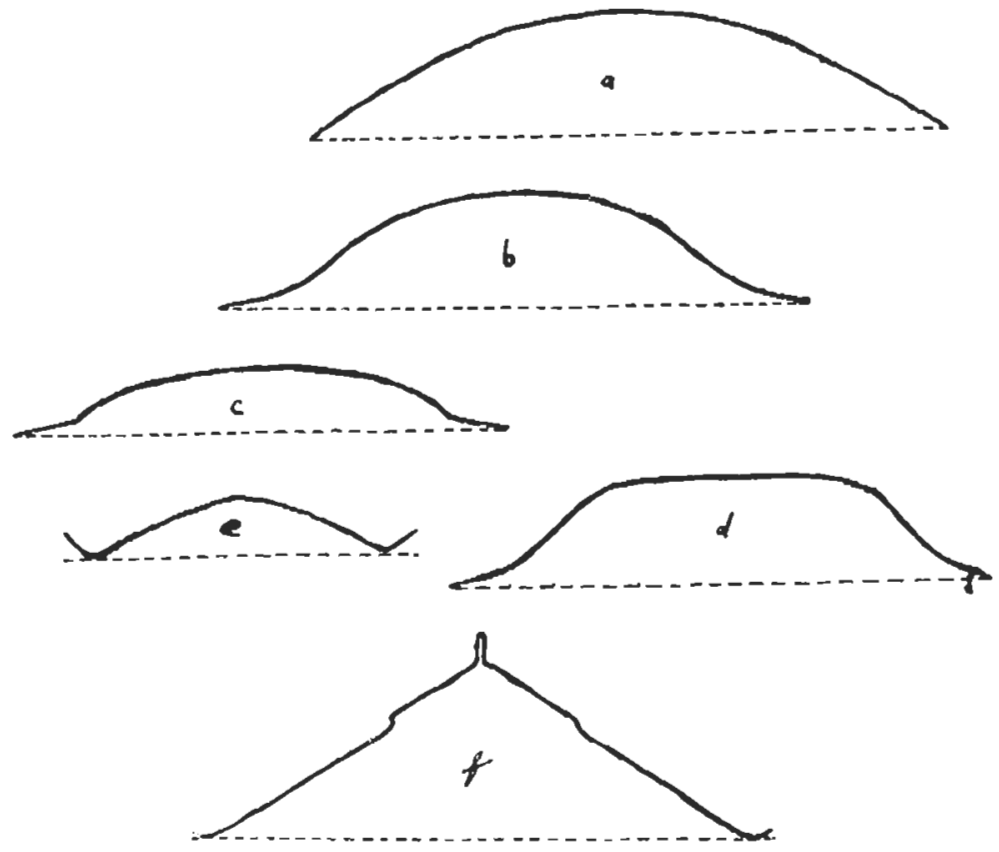

Fis, 2,-Exemples des formes de propil des bases des boucliers en métal. (a = Paris, Musćc de l'Armée, no. I 69\%9; b = Prague, Musée Naprstek, no. 4504; $\mathrm{c}=$ Praguc, Musée Naprstek, no. 19478; d = Prague, Musée Naprstek. no. $43604 ; \mathrm{c}=$ Paris, Musće de l'Homme, no. 43.27.349; $\mathrm{f}=$ Berne, Musée d'Hisioire, no. 926.)

calotte sphérique dont le bord remonte ${ }^{23}$ (Fig. 2e) et la forme conique avec le bord remontant légèrement (ce bouclier est pourvu d'un bouton ajouté sur l'umbo) ${ }^{24}$ (Fig. 2f).

${ }^{22}$ Vienne, Kunsthistorisches Museum, nos. C 85 et C 94.

"Paris, Musée de l'Homme, no. 43.27.349.

"Berne, Musée d'Histoire, no. 926. 
Observations métrologiques. - Le diamètre de la base des boucliers varie entre $255 \mathrm{~mm}^{25}$ et $530 \mathrm{~mm}^{26}$, mais le diamètre de la plupart des boucliers observés se classe entre 401 et $500 \mathrm{~mm}^{27}$.

La profondeur de la base varie entre $40 \mathrm{~mm}^{23}$ et $125 \mathrm{~mm}$, la plus grande étant celle du bouclier conique ${ }^{29}$. Mais c'est entre 51 et $80 \mathrm{~mm}$ que se situe la profondeur de la plupart des boucliers "

Quant à l'épaisseur de la base, elle est presque toujours de $1 \mathrm{~mm}$, dans un seul cas elle est de $2 \mathrm{~mm}{ }^{31}$ et dans un autre cas elle est même de $3 \mathrm{~mm}^{32}$.

Le poids des boucliers que nous avons pu examiner de ce point de vue varie entre $0,980 \mathrm{~g}^{33}$ et $3470 \mathrm{~g}^{34}$, mais sa plus grande fréquence est entre 1501 et $2000 \mathrm{~g}^{35}$.

Mécanisme qui permet de porter et de manier le bouclier.-Nous distinguons trois types de ce mécanisme, que nous appelons type à bossettes, type à poignée rivetée ou soudée et type à petits rivets.

${ }^{25}$ Paris, Musée de l'Homme, no. 43.27.349.

2.s Paris, Musée de l'Armée, no. I 6074.

${ }_{27}$ En répartissant les valeurs des diamètres dans les groupes d'une étendue de $50 \mathrm{~mm}$, nous obtenons les fréquences suivantes:

$\begin{array}{lllr}251-300 \mathrm{~mm} & 2 & 401-450 \mathrm{~mm} & 12 \\ 301-350 \mathrm{~mm} & 4 & 451-500 \mathrm{~mm} & 11 \\ 351-400 \mathrm{~mm} & 6 & 501-550 \mathrm{~mm} & 3\end{array}$

2* Berne, Musée d'Histoire, s. n.

20 Berne, Musée d'Histoire, no. 926.

31 En répartissant les valeurs des profondeurs dans les groupes d'une étendic de $10 \mathrm{~m} ı \mathrm{n}$, nous obtenons les fréquences suivantes:

$\begin{array}{lrrl}31-40 \mathrm{~mm} & 1 & 81-90 \mathrm{~mm} & 2 \\ 41-50 \mathrm{~mm} & 2 & 91-100 \mathrm{~mm} & 4 \\ 51-60 \mathrm{~mm} & 9 & 101-110 \mathrm{~mm} & 0 \\ 61-70 \mathrm{~mm} & 6 & 111-120 \mathrm{~mm} & 0 \\ 71-80 \mathrm{~mm} & 10 & 121-130 \mathrm{~mm} & 1\end{array}$

3 Paris, Musée de l'Armée, no. G 739.

${ }^{32}$ Berne, Musée d'Histoire, no. 965.

3. Prague, Musée Naprstek, no. 19478.

${ }^{34}$ Prague, Musée Naprstek, no. 4495.

${ }^{35}$ En répartissant les valeurs des poids dans les groupes d'une étendue de $500 \mathrm{~g}$, nous obtenons les fréquences suivantes:

$\begin{array}{rlll}501-1000 \mathrm{~g} & 1 & 2001-2500 \mathrm{~g} & 1 \\ 1001-1500 \mathrm{~g} & 4 & 2501-3000 \mathrm{~g} & 3 \\ 1501-2000 \mathrm{~g} & 8 & 3001-3500 \mathrm{~g} & 2\end{array}$


Type à bossettes.-Ce type est le plus répandu sur les boucliers que nous avons observés ${ }^{36}$ et son principe est le suivant: Les bossettes, quatre ou six, placées sur la surface externe, sont vissées par des vis ou attachées par des rivets qui traversent la base du bouclier sur la surface interne. Là, l'extrémité de la vis s'élargit et est trouée, formant ainsi un oeil. Les anneaux, passées par les yeux des vis, servent à maintenir les lanières, qui permettent de tenir et de manier le bouclier (Fig. 3).

FIG. 3.-Coupe d'un bouclier pourva du mécanisme à bossettes.

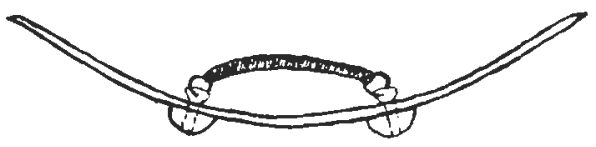

Les bossettes attachées sur la surface externe peuvent être de diverses formes, allant d'un hémisphère à une calotte très plate. Elles ont à l'intérieur un filetage, qui leur permet de servir d'écrou aux vis qui traversent la base. Exceptionnellement ${ }^{37}$, elles sont rivetées sur la base par un rivet massif, dont la tête est visible sur le sommet de la bossette.
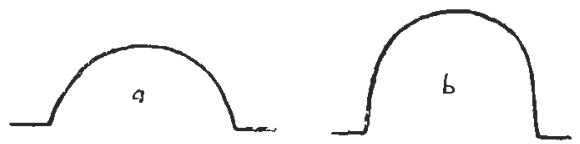
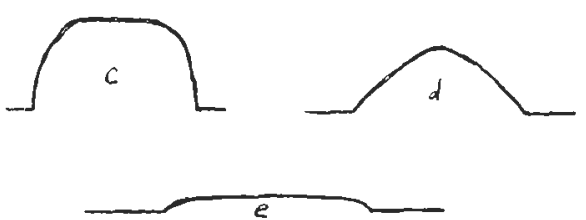

Fig. 4.-Coupes des bossettes de diverses formes. $(\mathrm{a}=$ Prague, $\mathrm{Mu}$ sée Naprstek, no. A 2680; b = Prague, Musée Naprstek, no. 4.505; c= Paris, Musée de l'Homme, no. 47.29.4; $\mathrm{d}=$ Berne, $\mathrm{Mu}-$ sée d'Histoire, no. 966; e $=\mathrm{Pa}$ ris, Musée de l'Armée, no. I 6074.)

Les boucliers observés sont presque tous pourvus de bossettes en acier ou éventuellement en fer, sur deux boucliers on trouve des bossettes en cuivre jaune $^{38}$ et sur deux autres en laiton, leur base étant, elle aussi, en laiton ${ }^{39}$.

36 Tous les boucliers que nous étudions sont pourvus du mécanisme de ce type, à l'exception des boucliers suivant: Prague, Musée Naprstek, no. 16 266; Paris, Musée de l'Homme, nos. 47.29.4 et 43.27.349; Vienne, Kunsthistorisches Museum, no. C 94.

${ }^{37}$ Berne, Musée d'Histoire, nos. 926, 965, 910 et 1426; Paris, Musée de l'Armée, no. I 6074.

${ }^{38}$ Berne, Musée d'Histoire, nos. 965 et 910.

${ }^{29}$ Berne, Musée d'Histoire, nos. 1370 et s. n. 
La forme des bossettes la plus répandue est celle d'un hémisphère (Fig. 4a), qui peut être parfois un peu prolongé (Fig. 4b) ou par contre un peu aplati sur le sommet (Fig. 4c). Sur un seul bouclier elles prennent la forme d'une calotte ${ }^{40}$ (Fig. 4 d) et sur un autre elles sont presque plates $^{41}$ (Fig. $4 \mathrm{e}$ ).

Le diamètre de la base des bossettes varie entre 27 et $35 \mathrm{~mm}$, leur hauteur entre 13 et $24 \mathrm{~mm}$. Autour de leur base se trouve une bordure
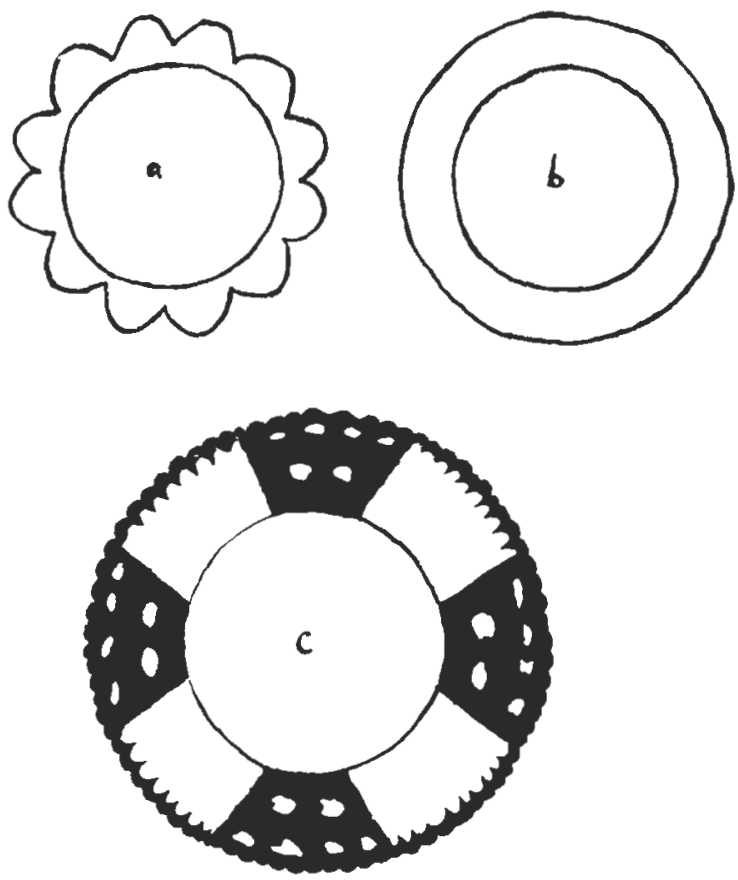

Fig. 5.-Formes des bordures des bossettes. (a = Prague, Musée Naprstek, no. A 2680; $\mathrm{b}=$ Paris, Musée de l'Armée, no. I 6670; $c=$ Paris, Musée de l'Armée, no. I 6074.)

qui est presque toujours polylobée (Fig. 5a) ou parfois circulaire ${ }^{42}$ (Fig. 5b). La bordure des bossettes qui sont presque plates est perforée (Fig. 5c). Certains boucliers ont des bossettes qui prennent des formes très décoratives ${ }^{43}$. Nous allons donner leur description dans le chapitre sur l'Analyse décorative.

4. Berne, Musée d'Histoire, no. 866.

4l Paris, Musée de l'Armée, no. I 6074.

${ }^{42}$ Paris, Musée de l'Armée, no. I 6670.

${ }^{43}$ Berne, Musée d'Histoire, nos. 965, 1426 et 1427. 
Le nombre des bossettes est de quatre, plus rarement de $\operatorname{six}^{44}$. Si elles sont quatre, elles sont alors placées autour de l'umbo sur les angles d'un carré imaginaire dont le côté varie entre 77 et $145 \mathrm{~mm}$, mais dans la plupart des cas il est de $75-90 \mathrm{~mm}$. Si les bossettes sont au nombre de six, alors quatre d'entr'elles sont aussi placées sur les angles d'un carré imaginaire autour de l'umbo, tandis que les deux autres se trouvent plus près du bord du bouclier, parallèlement avec un côté du carré imaginaire, à la distance de cca $80 \mathrm{~mm}$ de celui-ci.

Les vis ou rivets qui fixent les bossettes sont en fer et leur solidité est très variable. Ceci nous permet souvent de déterminer, à l'appui d'autres éléments, à quelle utilisation le bouclier était destiné.

F1G. 6.- Schéma de l'emplacement des lanières sur un bouclier à six bosselles.

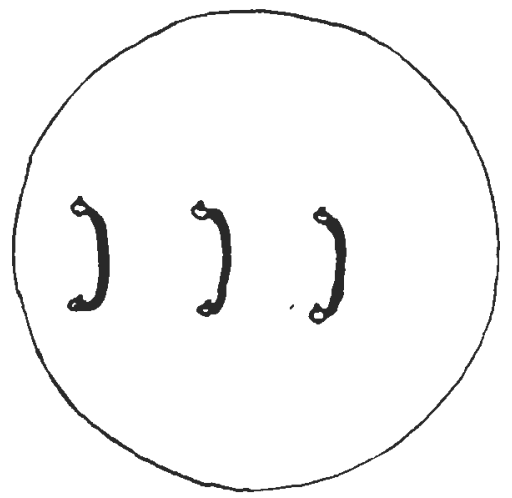

Les anneaux qui passent par les yeux des rivets sont en fil de fer enroulé, formant un cercle dont le diamètre se situe entre 9 et $30 \mathrm{~mm}$. L'épaisseur du fil de fer varie entre 1,5 et $5 \mathrm{~mm}$, ce qui fait que la solidité de ces anneaux est aussi très différente.

Les lanières sont généralement formées d'une bandelette en cuir, plus ou moins solide. Les lanières d'un bouclier observé sont en cuir double recouvert de velours beige ${ }^{45}$, un autre bouclier a des lanières faites de trois épaisseurs de cuir recouvertes de velours rouge ${ }^{46}$.

Les lanières sont au nombre de deux, sur les boucliers qui ont six bossettes elles sont au nombre de trois. Elles sont attachées parallèlement aux anneaux opposés (Fig. 6).

Sur un bouclier ${ }^{47}$, les lanières se croisent en diagonale et sont, au

* Prague, Musée Naprstek, no. 4506; Paris, Musée de l'Armée, no. G 739.

${ }^{45}$ Berne, Musée d'Histoire, no. 926.

to Berne, Musée d'Histoire, no. 910.

${ }^{47}$ Berne, Musée d'Histoire, no. 965. 
milieu, au point de croisement, attachées ensemble par un rivet, qui tient en plus un anneau (Fig. 7e) auquel est fixée encore une bandelette en cuir, dont la longueur est de $65 \mathrm{~mm}$. Cette bandelette porte, à son extrémité

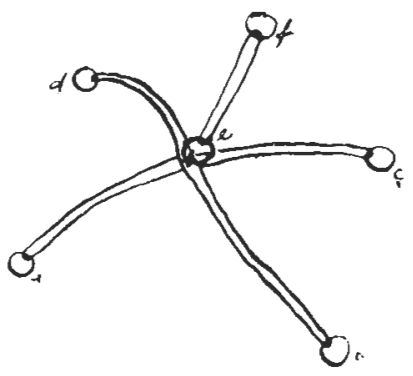

Frg. 7.-Schéma de la disposition des lanières en diagonale, avec une bandelette portant un anneau servant à la suspension du bouclier. (Berne, Musée d'Histoire, no. 965.)

opposée, un autre anneau (Fig. 7f), qui devait servir à la suspension du bouclier.

Mais nous observons que sur beaucoup de boucliers analysés les lanières manquent complètement, soit parce qu'elles ont disparu, soit parce que le bouclier n'en a jamais eu, n'étant qu'une pièce de décoration.

Type à poignée rivetée ou soudée ${ }^{48}$.- Ce type se distingue par une seule poignée en fer ou en cuivre jaune, en forme de tube coudé ou de canal (Fig. 8), qui est rivetée ou soudée sur la surface interne de la base.

FIG. 8.-.-Poignée en forme de canal. (Paris, Musée de l'Homme, no. 43.27.349.)

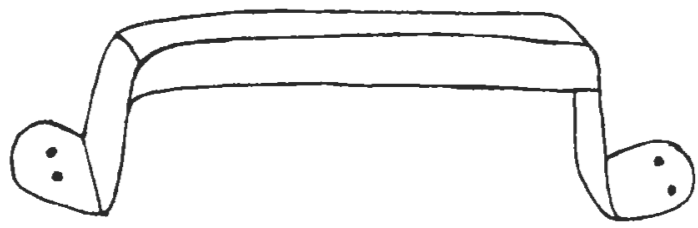

Type à petits rivets.-Un seul bouclier en métal possède ce type de mécanisme qui permet de le porter ${ }^{49}$. Ici, les rivets au nombre de dix sont retenus sur la surface externe par des plaques en forme de rosette et ils traversent la base du bouclier. Sur la surface interne, ils s'élargissent en formant une petite tête qui est trouée et par laquelle est passé un petit anneau. Les lanières manquent sur ce bouclier. La figure no. 9 nous montre la schéma de répartition des rivets sur la base du bouclier.

${ }^{48}$ Prague, Musée Naprstek, no. 16 266; Paris, Musée de l'Homme, no. 43.27.349; Vienne, Kunsthistorisches Museum, no. C 94.

${ }^{49}$ Vienne, Kunsthistorisches Museum, no. C 85. 
Éléments renforçant la solidité et l'efficacité du bouclier.-C'est une bordure rapportée, en métal, qui devait renforcer le bouclier contre le coup d'une arme de taille du côté de la tranche, ainsi qu'empêcher le glissement d'une arme offensive au-delà du bouclier. Elle est formée par

FIG. 9.-Schéma de réparition des rivets sur la base d'un bouclier pourvu du mécanisme qui permet de le porter, du lype «à pelits rivets». (Vienne, Kunsthistorisches Muscum, no. C 85.)

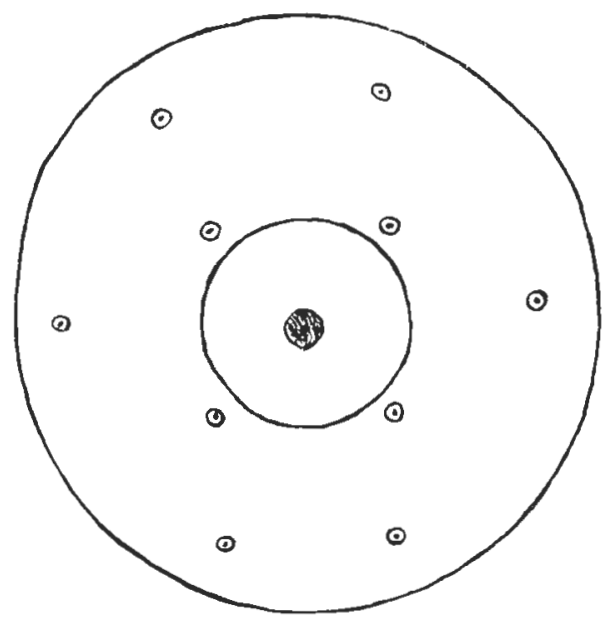

une bande en acier ou en cuivre jaune, demi-ronde, dont la largeur varie entre 5 et $10 \mathrm{~mm}$ et la hauteur entre 3 et $5 \mathrm{~mm}$. Elle est rivetée sur la circonférence extrême de la surface externe.

Trois des boucliers examinés n'ont pas cette bordure ${ }^{50}$. Un de ces boucliers ${ }^{51}$ renforce le bord de sa base en le faisant remonter et ainsi une bordure rapportée n'est plus nécessaire.

Les bossettes placées sur la surface externe, faisant partie du mécanisme permettant de porter le bouclier, pouvaient aussi empêcher ou au moins freiner le glissement d'une arme offensive au-delà du bord du bouclier.

Éléments amortissant les chocs.-C'est la matelassure et le coussinet qui forment ce groupe, les deux se trouvant sur la surface interne de la base.

La matelassure couvre entièrement la surface interne. Elle est formée de deux étoffes rondes superposées, entre lesquelles se trouve une

${ }^{50}$ Berne, Musée d'Histoire, no. 965; Paris, Musée de l'Armée, no. I 6074; Paris, Musée de l'Homme, no. 43.27.349.

${ }^{51}$ Paris, Musée de l'Homme, no, 43.27.349. 

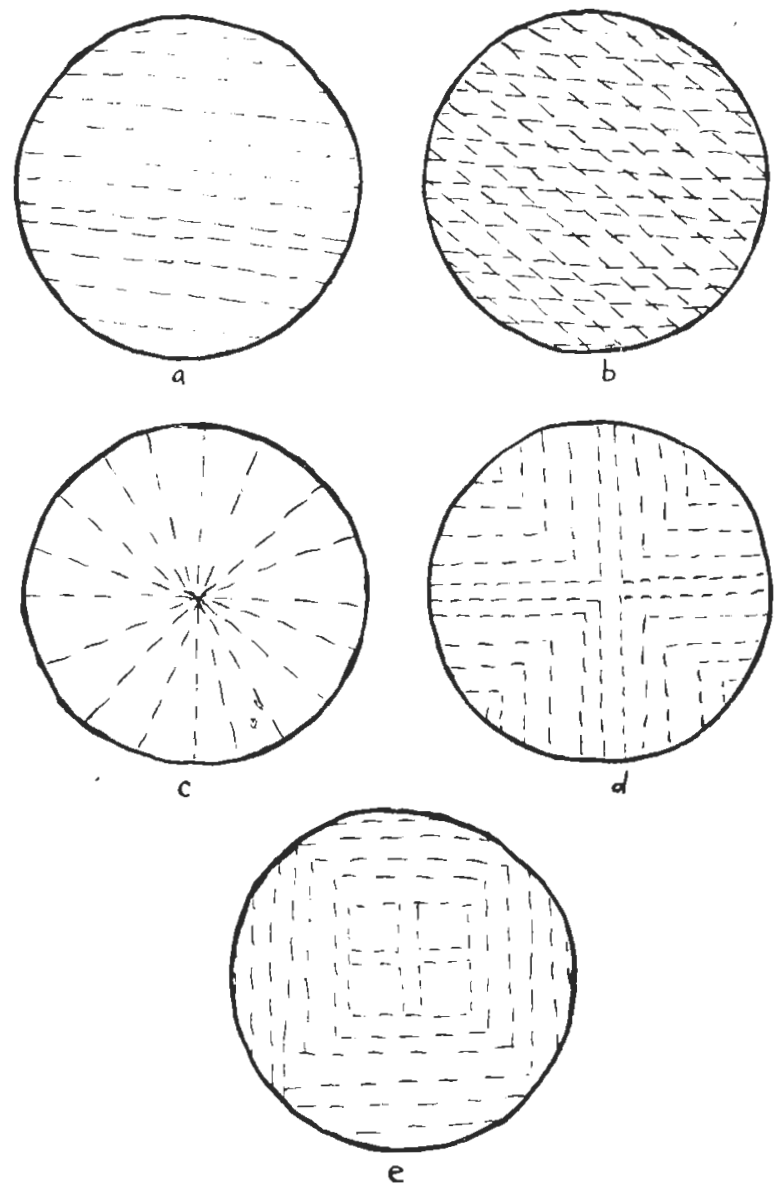

FIG. 10. - Types des arrangements des points de conture des matelassures.

mince couche de coton ou de laine crue. L'étoffe de dessous est habituellement une toile blanche ordinaire, celle de dessus est soit une toile rouge ordinaire, soit un velours, souvent rouge ou beige. Les deux étoffes sont cousues ensemble avec des points de couture dont l'arrangement varie d'un bouclier à l'autre. Nous pouvons distinguer les types suivants: des points en lignes parallèles ${ }^{52}$ (Fig. 10a), des points en lignes droites

${ }_{52}$ Prague, Musée Naprstek, no. 4505. 
croisées formant des losanges ${ }^{53}$ (Fig. 10b), des points en lignes radiées formant une étoile ${ }^{54}$ (Fig. 10c), des points en lignes à angle droit encastrées, qui sont réparties en quatre compartiments placés de chaque côté de deux axes perpendiculaires se coupant au centre du bouclier ${ }^{55}$ (Fig. 10d) et des points formant des carrés encastrés l'un dans l'autre, avec une croix au milieu du carré central ${ }^{56}$ (Fig. 10e).

La matelassure est collée par son étoffe de dessous sur la base du bouclier, un galon est parfois cousu ou collé sur son bord.

On ne trouve pas toujours une vraie matelassure mais il arrive qu'à sa place soient collés une simple toile ordinaire ${ }^{57}$ ou un cuir ${ }^{58}$. C'est alors de la «fausse» matelassure.

Sur l'umbo de la surface interne peut être placé un coussinet, qui est fixé par des vis ou rivets qui portent les lanières. Ces rivets traversent le coussinet dans ses angles. Le coussinet prend la forme d'un carré de 105 à $150 \mathrm{~mm}$ de côté, ou bien d'un rectangle de 125 à $190 \mathrm{~mm}$ de longueur et de 100 à $150 \mathrm{~mm}$ de largeur. Les coussinets rectangulaires sont utilisés surtout sur les boucliers qui ont six bossettes.

La couverture d'un coussinet est faite d'une étoffe en toile ou en velours, ou bien en cuir. La bordure des coussinets dont la couverture est en étoffe est souvent renforcée par une bande en cuir cousue. L'intérieur est rempli de coton ou de laine crue, ce qui donne au coussinet une épaisseur de 10 à $20 \mathrm{~mm}$ environ.

Souvent entre la tête de la vis et le coussinet il y a une plaque - rosette, passée par la vis, pour mieux fixer le coussinet.

On ne trouve pas de matelassure ou de coussinet sur tous les boucliers et leur qualité et leur solidité sont très variables.

\section{Boucliers en peau d'animal}

Pour son accessibilité et son élaboration relativement facile, la peau d'animal est l'un des matériaux le plus ancien qui servait à la fabrication de la base d'un bouclier. Et c'est grâce à ces avantages ainsi qu'à son poids assez léger qu'elle n'a, en principe, jamais cessée d'être utilisée.

53 Prague, Musée Naprstek, no. 43 604; Berne, Musée d'Histoire, no. 966; Paris, Musée de l'Homme, no. 62.71.29.

${ }^{5+}$ Berne, Musée d'Histoire, nos. 926 et 965.

${ }_{55}$ Prague, Musée Naptstek, nos. 19 478, 4 506, 16266 et 4504; Berne, Musée d'Histoire, nos. 910, 904, s. n. et Pers. 39; Paris, Musée de l'Armée, no. G 739; Paris, Musée de l'Homme, no. 34.141.2.

56 Prague, Musée Naprstek, no. 4453.

${ }^{57}$ Paris, Musée de l'Homme, no. 47.29.4.

${ }^{58}$ Prague, Musée Naprstek, no. 4507. 
Avant qu'on lui donne la forme désirée, la peau de l'animal devait être séchée pour qu'elle devienne suffisamment dure et solide ${ }^{59}$. La peau de rhinocéros pouvait être «au préalable bouillie dans l'huile et soumise à des procédés chimiques qui lui donnaient une belle transparence et une certaine élasticité nécessaire à son usage et à sa durée. La couleur de ces boucliers est ordinairement d'un jaune d'ocre, d'une nuance plus ou moins foncée. La teinte de celui-ci cst assez claire, et sa transparence est telle que le moindre corps opaque se distingue au travers» ${ }^{60}$. Le même procédé pouvait être fait avec la peau de buffle, mais sa translucidité et sa finesse restent inférieures à celles de la peau de rhinocéros (Fig. 24-25).

Cette préparation de la peau une fois terminée, la peau est coupée et moulée suivant la forme et le profilage désirés. Les boucliers en peau d'animal résistent aux coups de sabre et de flèche et certains d'entre eux, dit-on, même à ceux d'une balle ${ }^{61}$.

Les espèces de peaux utilisées pour la base des boucliers observés par nous sont la peau de rhinocéros qui peut être translucide ${ }^{62}$ ou non translucide ${ }^{63}$, la peau de buffle qui peut être également translucide ${ }^{64}$ ou non translucide ${ }^{65}$, la peau d'éléphant ${ }^{6 /}$ et dans un cas une peau que nous n'avons pas été en mesure de déterminer ${ }^{67}$. La base d'un des boucliers observés est faite d'une plaque de bois qui est recouverte de chaque côté par une peau de buffle.

Construction de la base.-Toutes les bases en peau des boucliers examinés sont fabriquées avec une seule peau, préparée comme nous l'avons décrit ci-dessus, à l'exception du bouclier où entre deux peaux de buffle est interposée une plaque en bois ${ }^{68}$. Ces trois parties, dont cha-

${ }^{99}$ Voir Berthold Laufer: Chinese Clay Figures, part I, Prolegomena on the History of Defensive Armor, Chicago, 1914, p. 81.

"o Floriant Gille: Muscé de Tzarskoe-Selo, ou collection d'armes de Sa Majesté l'Empereur de toutes les Russies, t. I, St. Pétersbourg \& Carlsruhe, 1835, texte se rapportant à la planche LXXIV.

" Voir Joseph Skejton: Engraved Illustrations of Ancient Arms and Armour, from the Collection of Llewelyn Meyrick, at Goodrich Court, Herefordsbire, t. II, Oxford, 1830 , texte se rapportant à la planche CXLI.

${ }_{62}$ Berne, Musée d'Histoire, nos. 969, 967, 970, 980 et 1212; Paris, Musée de l'Armée, nos. I 97', I $97^{2}$ et s. n.; Paris, Musée de l'Homme, nos, 32.35 .12 et 32.35.30.

${ }^{\circ 3}$ Paris, Musée de l'Homme, nos. 35.115.72, 35.115.524 et 35.115.70.

${ }^{64}$ Berne, Musée d'Histoire, nos. 921 et 968; Paris, Musée de l'Homme, no. 46.38.170.

${ }_{65}$ Berne, Musée d'Histoire, nos, 973, 971, 972, 1213 et 974; Paris, Musée de l'Homme, no. X.49.5.

to Berne, Musée d'Histoire, no. 975

67 Vienne, Historisches Museum der Stadt Wien, no. 126.229.

${ }^{68}$ Paris, Musée de l'Homme, no. 43.27.350. 
cune a une épaisseur de cca 1,5 mm, sont collées ensemble et ce n'est qu'après une observation attentive qu'on peut s'apercevoir que la base n'est par uniquement en peau.
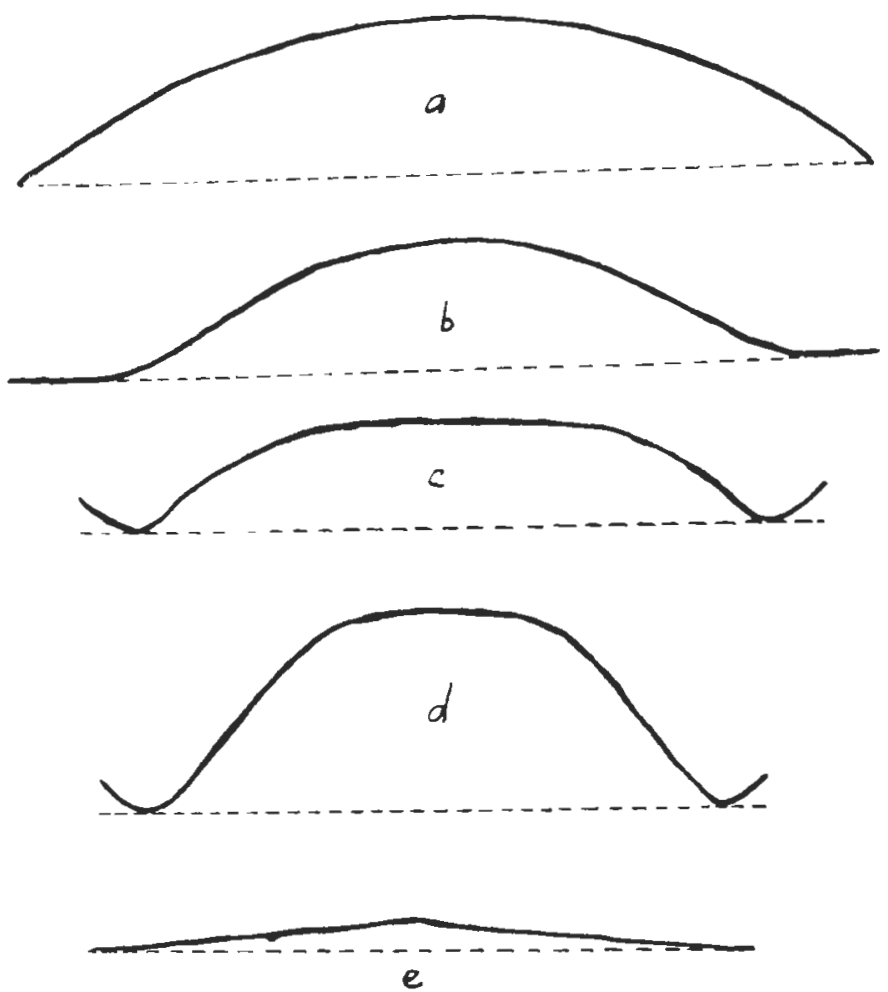

FiG. 11.-Exemples des formes de profil des bases des boucliers en pecu d'animal. ( $\mathrm{a}=$ Vienne, Historisches Museum der Stadt Wien, no. $126.229 ; b=$ Berne, Musée d'Histoire, no. 968; $c=$ Berne, Musée d'Histoire, no. 972; d = Paris, Musée de l'Armée, sans no.; $\mathrm{e}=$ Berne, Musée d'Histoire, no. 1213.)

La base circulaire des boucliers peut obtenir, par moulage, diverses formes bombées qui sont, approximativement, les suivantes (Fig. 11): La forme d'une calotte sphérique ${ }^{69}$ (Fig. 11a), la forme d'une calotte

*9 Berne, Musée d'Histoire, nos. 971 et 975; Paris, Musće de l'Homme, nos. 35.115.72 et 35.115.524; Vienne, Historisches Museum der Stadt Wien, no. 126.229. 
sphérique aplatie sur le bord ${ }^{70}$ (Fig. 11b), la forme d'une calotte sphérique aplatie dont le bord remonte ${ }^{71}$ (Fig. 11c), la forme d'une calotte sphérique dont le bord remonte ${ }^{72}$ (Fig. 11d) et dans un seul cas on observe la forme conoïde très aplatie ${ }^{73}$ (Fig. 11e).

Le diamètre de la base varie entre $260 \mathrm{~mm}^{74}$ et $580 \mathrm{~mm}^{75}$, mais il se situe le plus souvent entre 401 et $450 \mathrm{~mm}^{76}$. La profondeur de la base est très variée, la plus petite est de $20 \mathrm{~mm}^{\pi}$ tandis que la plus grande est de $132 \mathrm{~mm}^{78}$. On peut dire que la profondeur la plus courante est entre 61 et $70 \mathrm{~mm}^{79}$. L'épaisseur de la base varie entre 3 et $10 \mathrm{~mm}$, mais la plupart des bases ont une épaisseur de $5 \mathrm{~mm}$. Le poids oscille entre $900 \mathrm{~g}^{80}$ et $2820 \mathrm{~g}^{81}$ et sa variabilité ne nous permet pas de déterminer les limites de l'oscillation la plus fréquente ${ }^{82}$.

${ }^{70}$ Berne, Musće d'Histoire, nos. 1212, 921, 968, 973 et 974; Paris, Musée de l'Armée, nos. I 97' et I 97²; Paris, Musée de l'Homme, nos. 46.38.170 et 35.115.70.

${ }^{71}$ Berne, Musée d'Histoire, no. 970; Paris, Musée de l'Armée, sans no.; Paris, Muséc de l'Homme, nos. 32.35.12 et X.49.5.

${ }^{72}$ Berne, Musée d'Histoire, nos. 969, 967, 980 et 972; Paris, Musée de l'Homme, nos. 32.35.30 et 43.27.350.

is Berne, Musée d'Histoire, no. 1213.

74 Berne, Muséc d'Histoire, no. 1212.

${ }_{75}$ Berne, Musće d'Histoire, no. 968.

${ }^{76}$ En répartissant les valeurs des diamètres dans les groupes d'une étendue de $50 \mathrm{~mm}$, nous obtenons les fréquences suivantes:

$\begin{array}{lrll}251-300 \mathrm{~mm} & 3 & 451-500 \mathrm{~mm} & 3 \\ 301-350 \mathrm{~mm} & 3 & 501-550 \mathrm{~mm} & 2 \\ 351-400 \mathrm{~mm} & 2 & 551-600 \mathrm{~mm} & 2 \\ 401-450 \mathrm{~mm} & 10 & & \end{array}$

7 Berne, Musée d'Histoire, no. 1213.

${ }^{78}$ Paris, Musée de l'Armée, sans no.; Paris, Musée de l'Homme, no. 32.35.12.

${ }^{79}$ En répartissant les valeurs des profondeurs dans les groupes d'une étendue de $10 \mathrm{~mm}$, nous obtenons les fréquences suivantes:

$\begin{array}{llrl}11-20 \mathrm{~mm} & 1 & 81-90 \mathrm{~mm} & 3 \\ 21-30 \mathrm{~mm} & 1 & 91-100 \mathrm{~mm} & 1 \\ 31-40 \mathrm{~mm} & 1 & 101-110 \mathrm{~mm} & 1 \\ 41-50 \mathrm{~mm} & 3 & 111-120 \mathrm{~mm} & 0 \\ 51-60 \mathrm{~mm} & 3 & 121-130 \mathrm{~mm} & 1 \\ 61-70 \mathrm{~mm} & 6 & 131-140 \mathrm{~mm} & 2 \\ 71-80 \mathrm{~mm} & 2 & & \end{array}$

${ }^{80}$ Berne, Musée d'Histoire, no. 974.

${ }^{81}$ Berne, Musée d'Histoire, no. 968.

${ }^{82}$ En répartissant les valeurs des poids dans les groupes d'une étendue de $500 \mathrm{~g}$, nous obtenons les fréquences suivantes:

$\begin{array}{rlll}501-1000 \mathrm{~g} & 2 & 2001-2500 \mathrm{~g} & 2 \\ 1001-1500 \mathrm{~g} & 2 & 2501.3000 \mathrm{~g} & 1 \\ 1501-2000 \mathrm{~g} & 2 & \end{array}$


Mécanisme qui permet de porter et de manier le bouclier.-Nous ne trouvons qu'un seul type de ce mécanisme, c'est celui à bossettes. Un seul bouclier ${ }^{83}$ n'est pourvu d'aucun mécanisme qui permettrait de le porter, cet objet n'était pas destiné au combat mais il s'agit d'une pièce décorative.

Les bossettes sont au nombre de quatre ou six, et elles sont toujours attachées par un rivet qui traverse la base du bouclier. La tête du rivet est toujours visible sur le sommet des bossettes.

Le matériau utilisé pour la fabrication des bossettes est en général le fer ou l'acier, mais nous trouvons aussi des bossettes en cuivre jaune ${ }^{84}$ ou en argent ${ }^{85}$.

La forme des bossettes la plus répandue est celle d'une calotte, qui est parfois assez plate (Fig. 12c), on observe aussi un calice ${ }^{86}$, un cône ${ }^{87}$
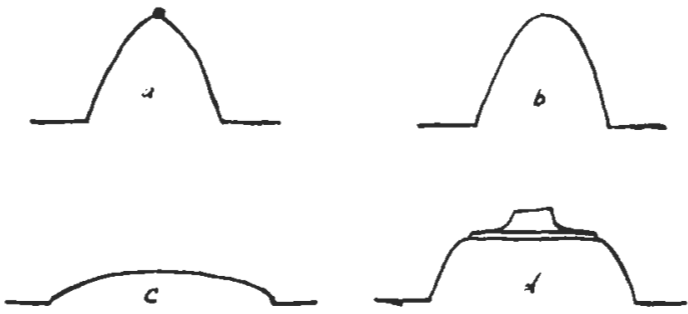

FIG 12.-Formes des bossettes des boucliers en peau d'animal, présentées en coupe. (a = Paris, Musée de l'Homme, no. 46.38.170; $b=$ Berne, Musée d'Histoire, no. 921; c = Paris, Musée de l'Homme, no. 35.

115.524; d = Berne, Musée d'Histoire, no. 1213.)

(Fig. 12a) qui peut être arrondi sur le sommet ${ }^{88}$ (Fig. 12b), ou bien dans un cas aussi un segment sphérique au sommet duquel est ajouté un bouton $^{89}$ (Fig. 12d),

Le diamètre de la base des bossettes varie entre 24 et $50 \mathrm{~mm}$, leur hauteur varie entre 7 et $30 \mathrm{~mm}$. La bordure autour de la base des bossettes prend souvent des formes assez décoratives.

${ }^{23}$ Paris, Musée de l'Armée, sans no.

\&t Berne, Muscé d'Histoire, no. 974; Paris, Musée de l'Homme, no. 46.38.170; Vienne, Historisches Museum der Stadt Wien, no. 126.229.

${ }^{85}$ Berne, Musée d'Histoire, nos. 973 et 971.

${ }^{86}$ Berne, Musée d'Histoire, nos. 969 et 1212; Paris, Musée de l'Armée, no. I 97'.

${ }^{87}$ Berne, Musée d'Histoire, no. 970.

${ }^{88}$ Paris, Musée de l'Homme, no. 46.38.170.

${ }^{89}$ Berne, Musée d'Histoire, no. 1213. 
Quand les bossettes sont au nombre de quatre, elles sont placées autour de l'umbo sur les angles d'un carré imaginaire de 87 à $140 \mathrm{~mm}$ de côté, mais le plus souvent de 100 à $123 \mathrm{~mm}$. Quand les bossettes sont au nombre de $\operatorname{six}^{90}$, quatre d'entre elles sont alors, elles aussi, placées sur les angles d'un carré imaginaire et les deux qui restent se trouvent à $80-90 \mathrm{~mm}$ d'un côté de ce carré imaginaire, parallèlement à lui.

On voit sur un des boucliers examinés cinq bossettes, dont quatre sont placées sur les angles d'un carré imaginaire dont le côté est de $140 \mathrm{~mm}$ et la cinquième se trouve au milieu de ce carré, sur l'umbo. Les rivets sur lesquels ces bossettes sont attachées ne correspondent pas aux rivets qui tiennent les anneaux d'attachement des lanières sur la surface interne ${ }^{91}$.

Les rivets qui traversent la base sur la surface interne s'élargissent dès qu'ils sortent sur cette surface. Cette partie élargie, qui est trouée d'un oeil dans lequel est passé un anneau, peut prendre diverses formes, dont nous pouvons observer des exemples sur la figure no. 13.
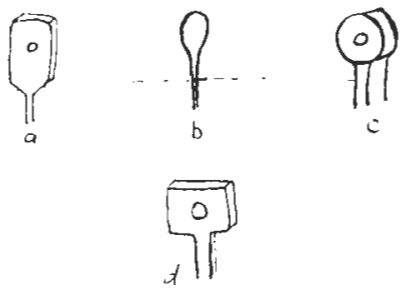

FIg. 13.-Exemples des types de têtes de rivets. ( $\mathrm{a}=$ Berne, Musće d'Histoire, no. 1213; $\mathrm{b}=$ Berne, Musée d'Histoire, no. 974; $\mathrm{c}=$ Berne, Musée d'Histoire, no. 975; $\mathrm{d}=$ Paris, Musée de l'Armée, no. I $97^{1}$.)

Les anneaux qui sont passés par les têtes de rivets sont en fil de fer ou d'acier, dans un seul cas en cuivre jaune ${ }^{92}$. Ils forment un cercle dont le diamètre varie entre 25 et $35 \mathrm{~mm}$ et ils sont presque toujours très solides.

Les lanières sont généralement formées, comme nous l'avons vu aussi sur les boucliers en métal, d'une bandelette en cuir qui passe par les anneaux. Après avoir été passées par les anneaux, leurs deux extrémités sont courbées et cousues avec le reste de la bandelette et c'est par cette façon que les bandelettes sont fixées (Fig. 14).

Les lanières en cuir double ou triple, recouvertes de velours rouge, vert ou beige ${ }^{93}$ sont plus solides et plus décoratives que les bandelettes

* Berne, Musée d'Histoire, nos. 969, 970, 921, 968 et 971; Paris, Musée de l'Armée, no. I 972; Vienne, Historisches Museum der Stadt Wien, no. 126.229.

${ }_{91}$ Berne, Musée d'Histoire, no. 971.

92 Berne, Musée d'Histoire, no. 974.

93 Berne, Musée d'Histoire, no. 921; Paris, Musée de l'Homme, nos. 32.35.12, 32.35.30 et X.49.5. 
en cuir. Mais on peut voir aussi des lanières formées d'une longue bandelette de cuir tressée, les extrémités de ces lanières sont aussi fixées aux anneaux par tressage ${ }^{94}$ (Fig. 15).

Les lanières sont au nombre de deux, mais sur les boucliers qui ont

FIG. 14.-Schéma de fixation d'une bandeletle sur un anneau. (Paris, Musée de l'Homme, no. 46.38.170.)

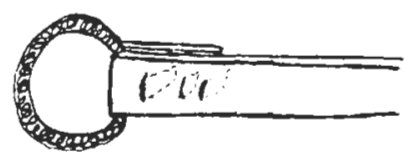

six bossettes elles sont au nombre de trois. Elles sont fixées parallèlement aux anneaux opposés, à quelques exceptions près ${ }^{95}$ où elles sont placées en diagonale.

Les boucliers en peau d'animal sont pourvus parfois d'une bretelle ${ }^{96}$ quí permet de porter le bouclier sur le dos ou sur l'épaule. C'est une longue bande de cuir d'une longueur très variable (entre 200 et $1800 \mathrm{~mm}$ ), une boucle fixée à une de ses extrémités permet de régler sa

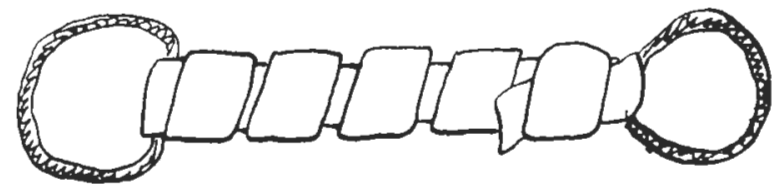

FIg. 15.-Lanière formée par une bande de cuir tressée. (Paris, Musée de l'Homme, no. 35.115.524.)

longueur suivant les besoins. Elle est attachée sur certains des anneaux sur lesquels sont fixées déjà les lanières (Fig. 16).

Éléments amortissant les chocs.-Sur les boucliers en peau d'animal c'est un coussinet qui protège la main tenant le bouclier contre les chocs. Il est fixć sur l'umbo de la surface interne, entre les lanières, et la plupart des boucliers que nous avons examinés le possèdent ${ }^{97}$.

La plupart des coussinets prend la forme d'un carré, dont le côté varie entre 105 et $150 \mathrm{~mm}$. Certains de ces coussinets en forme de carré ont leurs côtés découpés, formant ainsi une sorte d'étoile à quatre pointes (Fig. 17b). Les boucliers qui sont à six bossettes ont leur coussinet en

${ }^{94}$ Paris, Musée de l'Homme, no. 46.38.170.

${ }_{95}$ Berne, Musée d'Histoirc, no. 974; Paris, Musée de l'Homme, no. 46.38.170.

\% Berne, Musée d'Histoire, nos. 969, 971 et 975; Paris Musée de l'Homme, nos. 46.38.170 et 35.115.524.

"Dans notre «population», 18 sur 25 boucliers en peau d'animal possèdent un coussinet. 

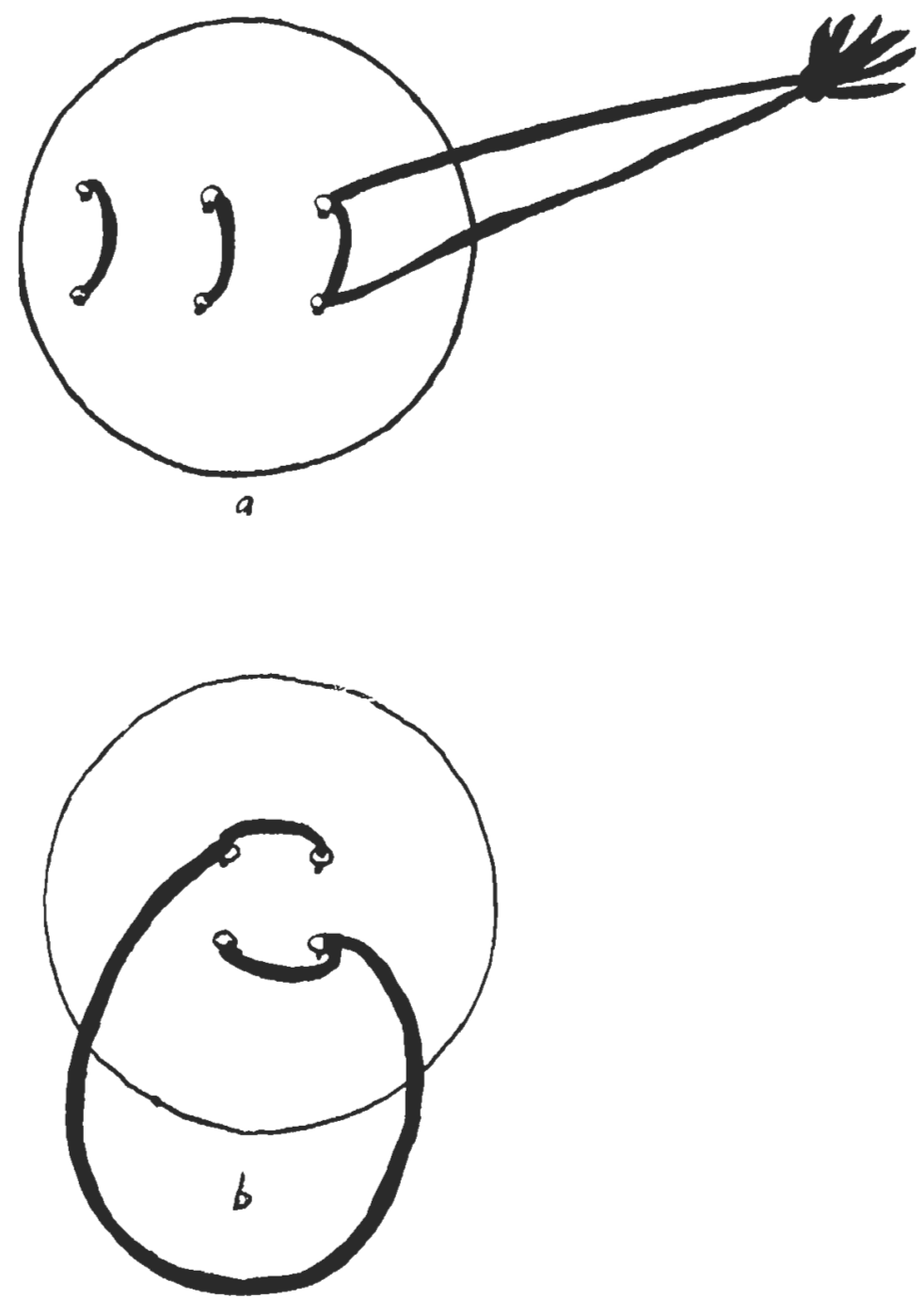

FIG. 16.-Exemples de l'emplacement des bretelles. (a = Berne, Musée d'Histoire, no. 969; b = Paris, Musée de l'Homme, no. 35.115.524.) 
forme de rectangle, dont l'un des petits côtés peut être pointu ${ }^{98}$, dentele ${ }^{99}$, ou découpé en dents de scie ${ }^{100}$ (Fig. 17a). Dans un seul cas un bouclier à six bossettes est pourvu d'un coussinet en forme de carré ${ }^{101}$.

FIG. 17.-Exemples des formes de conssinets. ( $\mathrm{a}=$ Paris, Musée de l'Armée, no. I $97^{2} ; b=$ Paris, Musée de l'Homme, no. 35.115.70.)
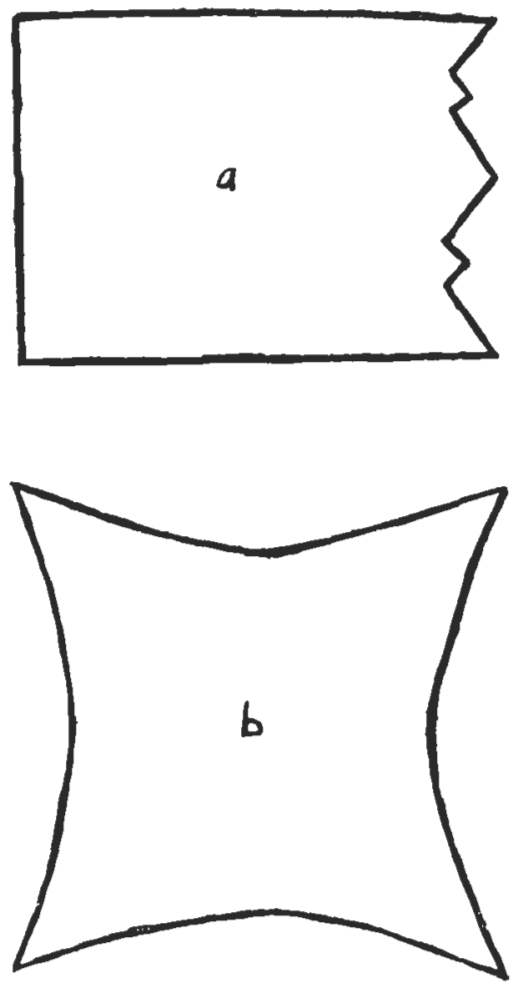

Un des boucliers observés, qui est à quatre bossettes, a son coussinet en forme circulaire ${ }^{102}$, il s'agit d'une exception.

Les coussinets sont fabriqués soit en cuir, soit en velours (au moins pour la partie supérieure), et souvent les deux moitiés de la taie sont cousues ensemble sur les bords avec une bande (cca $5 \mathrm{~mm}$ large) en
${ }^{98}$ Berne, Musée d'Histoire, no. 921.
"Berne, Musée d'Histoire, no. 969.
${ }^{100}$ Paris, Musée de l'Armée, no. I $97^{2}$.
${ }^{101}$ Berne, Musée d'Histoire, no. 970.
${ }^{102}$ Berne, Musée d'Histoire, no. 967. 
grosse toile ou en cuir. L'intérieur des coussinets est rempli de coton brut, ce que leur donne une épaisseur allant de 20 à $30 \mathrm{~mm}$.

Les coussinets sont fixés sur la base par quatre rivets centraux qui portent les lanières et qui traversent les coussinets dans leurs angles. Le coussinet rond est placé entre les rivets et il est attaché à eux à l'aide d'oeillets en fil de coton.

\section{Boucliers en osier tressé}

Nous comprenons dans ce groupe les boucliers dont la base est, dans sa plus grande partie, fabriquée en tiges de jonc, en tiges de canne ou en rameaux de figuier, qui sont entoulés concentriquement et tressés de fils de soie, de coton, ou d'or et d'argent. Il nous était impossible de distinguer visuellement les espèces de bois utilisées, surtout quand celui-ci est



FıG. 18.-Coupe d'un bouclier en osier. (1. Partie de la base en osier tressé; 2. Disque central en acier formant l'umbo; 3. Rondelle en gros tissu; 4. Morceau de bois posé sous le disque central.)

presque entièrement recouvert de fils tressés. Ici seulement un examen fait par un spécialiste en bois, sans doute à l'aide d'une analyse chimique, pourrait décider univoquement de l'espèce utilisée. Comme cette collaboration ne nous était pas possible, nous ne sommes pas en mesure de faire une distinction et nous nous limiterons à constater que ces boucliers sont en osier tressé, utilisant le terme osier comme terme général ${ }^{103}$, car c'est le bois le plus répandu pour ce genre de travail et il est possible que certains des boucliers examinés étaient effectivement faits en osier (Fig. 26).

Construction de la base.-Les rameaux de l'osier, dont le diamètre est de 5 à $8 \mathrm{~mm}$, sont disposés concentriquement, les uns à côté des autres. Les fils passant une fois dessus et une fois dessous et d'autres en sens inverse les lient très fermement ensemble formant ainsi une surface cohérente.

${ }^{103}$ Nous trouvons dans PAul-Émile LitTré: Dictionnaire de la langue française, t. III, Monte-Carlo, 1968, p. 4349, la définition suivante de I'osier: «1. Arbrisseau dont les jets sont très pliants»...; «2. Jet de cet arbrisseau»... 
L'umbo en acier, en forme de calotte parfois légèrement aplatie, est fixé sur le bord intérieur de la partie de la base en osier par quatre rivets dont les têtes prennent souvent la forme d'une rosette.

Sous ce disque, l'espace est rempli par un morceau de bois, rond ou
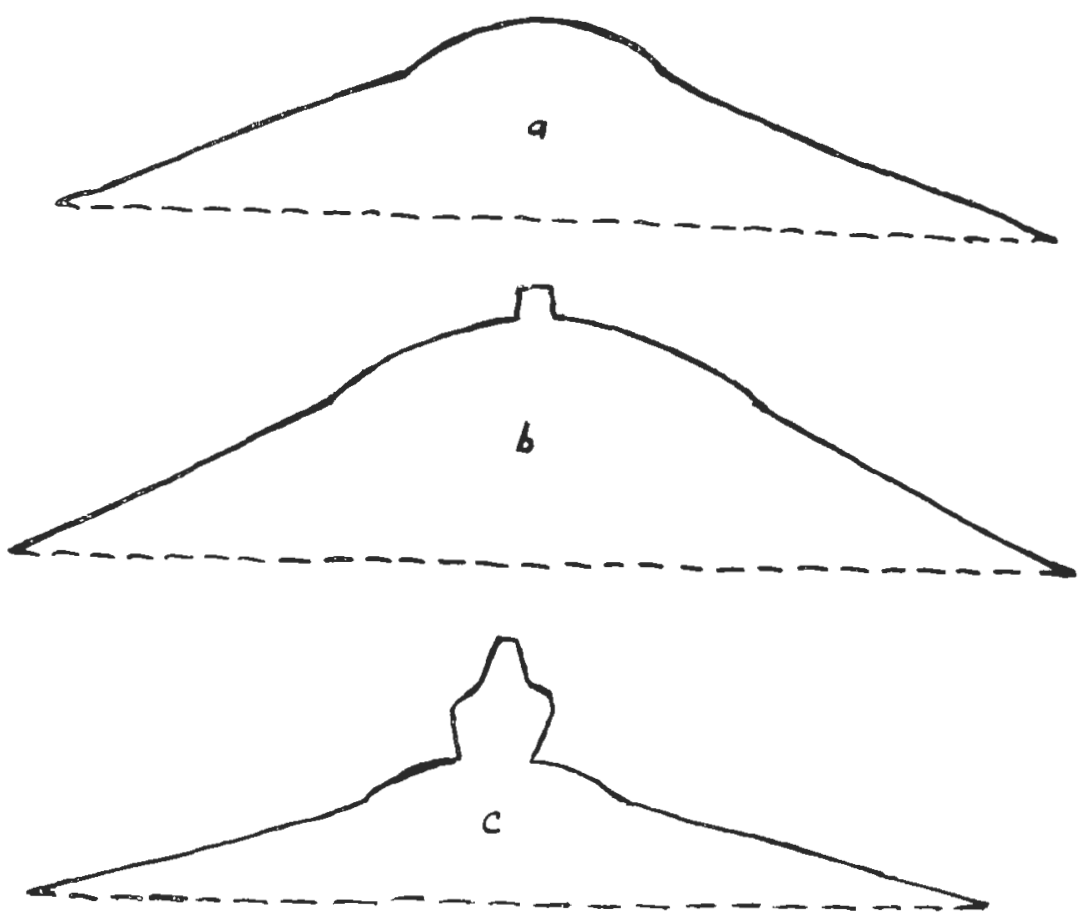

Fic. 19.-Exemples de profil des boucliers en osier tressé. (a = Paris, Musée de l'Homme, no. 32.35.10; $b=$ Paris, Musée de l'Armée, no. I 89; $c=$ Paris, Musée de l'Homme, no. 32.35.9.)

carré. L'umbo en acier est souvent posé sur une rondelle en gros tissu dont le bord dépasse le bord de l'umbo (Fig. 18).

Ces boucliers sont très légers et très résistants aux armes de taille ainsi qu'aux flèches ${ }^{104}$, néanmoins on observe parfois sur des miniatures

1:H À propos des boucliers en figuier Di Marsigl dit: «on les fait le plus souvent de figuier, parcequ'outre que ce bois est léger, il est aussi fort liant, \& propre à parer les coups d'estoc \& de taille, on les couvre de peaux, \& très souvent de cordes de coton, ce qui ne les rend pas plus pesans». ConTe di Marsigli: L'Étut militaire de l'Empire Otloman, t. II, La Haye, Amsterdam, 1732, p. 8. 
un bouclier en osier coupé en deux ${ }^{105}$, ce qui pouvait être probablement provoqué par le coup d'une arme de taille venant contre la tranche du bouclier.

En principe, la forme de la base des boucliers étudiés ne varie pas pour sa partie en bois, où elle prend une forme conoïde. L'umbo prend la forme d'une calotte qui peut être légèrement aplatie, son sommet peut être pourvu d'une pointe saillante ${ }^{16 s}$. En général, nous pouvons alors constater que la forme des boucliers en osier est conoïde, arrondie en calotte sur le sommet (Fig. 19).

Le diamètre des bases des boucliers en osier tressé varie entre $485^{107}$ et $620 \mathrm{~mm}{ }^{i 08}$, mais il se situe le plus souvent entre 501 et $600 \mathrm{~mm}^{109}$. La profondeur de ces boucliers oscille entre $75^{110}$ et $140 \mathrm{~mm}^{111}$, elle est souvent assez grande, entre 121 et $140 \mathrm{~mm}^{112}$. Quant à l'épaisseur, elle est, pour la partie en osier, entre 5 et $8 \mathrm{~mm}$, pour la partie en métal entre 1 et $2 \mathrm{~mm}$. Nous n'avoins pal, pour des raisons techniques, peser aucun des boucliers en osier. A titre d'information nous donnons ici le poids d'un bouclier ${ }^{113}$, que nous avons trcuvé dans le fichier du musée. Il est de $1800 \mathrm{~g}$ pour le diamètre de $598 \mathrm{~mm}$.

Mécanisme permetlant de porter et de manier le bouclier.-Tous les boucliers sont pourvus de ce mécanisme, qui est du type à petits rivets. Les rivets, au nombre de six à quatorze, retenus sur la surface externe par des plaques circulaires ou de diverses formes décoratives, traversent la base du bouclier, s'élargissent sur la surface externe formant soit des

${ }^{105}$ Voir par exemple une miniature du Săhnäme, qui date de 1440 environ, reproduite dans Basil Gray: La peinture persane, Genève, 1961, p. 91.

${ }^{106}$ Paris, Musée de l'Armée, no. I 89; Paris, Musée de l'Homme, no. 32.35.9.

107 Paris, Musée de l'Armée, no. I 91.

108 Paris, Musée de l'Armée, no. I 89.

10. En répartissant les valeurs des diamètres dans les groupes d'une étendue de $50 \mathrm{~mm}$, nous obtenons les fréquences suivantes:

$\begin{array}{llll}451-500 \mathrm{~mm} & 1 & 551-600 \mathrm{~mm} & 4 \\ 501-550 \mathrm{~mm} & 4 & 601-650 \mathrm{~mm} & 2\end{array}$

110 Paris, Musée de l'Armée, no. I 91.

"11 Paris, Musée de l'Armée, no. I 89.

112 En répartissant les valeurs des profondeurs dans les groupes d'une étendue de $10 \mathrm{~mm}$, nous obtenons les fréquences suivantes:

$\begin{array}{rrrr}71-80 \mathrm{~mm} & 1 & 111-120 \mathrm{~mm} & 0 \\ 81-90 \mathrm{~mm} & 1 & 121-130 \mathrm{~mm} & 2 \\ 91-100 \mathrm{~mm} & 1 & 131-140 \mathrm{~mm} & 2 \\ 101-110 \mathrm{~mm} & 0 & & \end{array}$

${ }^{113}$ Vienne, Kunsthistorisches Museum, no. C 172. 
oeillets assez larges pour y faire passer les lanières, soit des oeillets plus petits par lesquels sont passés les anneaux qui portent les lanières.

Les petites plaques qui tiennent les rivets sont en acier ${ }^{114}$, en bron$z^{115}$ ou en cuivre jaune. Elles ont une forme circulaire ${ }^{116}$ (Fig. 20a),

FIG. 20.--Types des plaques tenant les rivets. (a = Paris,
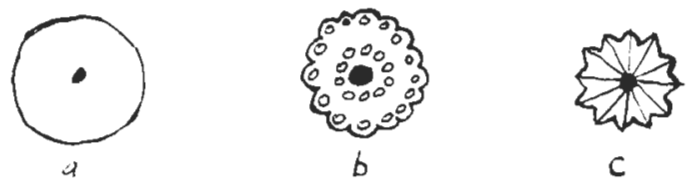

Musée de l'Armée, no. I 91;

$\mathrm{b}=$ Paris, Musée de l'Hom-

me, no. $32.35 .10 ; \mathrm{c}=\mathrm{Pa}-$

ris, Musée de l'Armée, no.

I 87; $\mathrm{d}=$ Paris, Musée de

l'Homme, no. 32.35.9.)

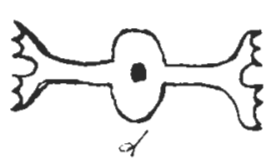

polylobée ${ }^{117}$ (Fig. 20b), ou bien, comme on observe sur un bouclier ${ }^{118}$, la forme d'une bande en bronze prolongée, arrondie au centre et dentelée aux extrémités élargies (Fig. 20d). Elles peuvent aussi avoir la forme d'une étoile ${ }^{119}$ (Fig. 20c). Leurs diamètres varient entre 14 et $38 \mathrm{~mm}$. Les mêmes plaques sont généralement placées aussi sur la surface interne, traversée par les rivets dès qu'ils sortent de la base et aidant à renforcer leur stabilité.

Les rivets sont généralement en acier, mais on les trouve aussi en bronze ${ }^{120}$. Leur élargissement sur la surface interne prend une forme ronde avec le diamètre allant de 5 à $12 \mathrm{~mm}$, ou bien la forme d'un cristal ${ }^{121}$. Quatre de ces rivets sont toujours placés autour de l'umbo en acier, aux angles d'un carré imaginaire dont les côtés varient entre 140 et $200 \mathrm{~mm}$. D'autres rivets sont placés d'une façon irrégulière, la figure no. 21 nous montre quelques exemples de leur position.

Les anneaux qui passent par certains rivets sur la surface interne sont en fer ou en acier, en forme circulaire avec un diamètre allant de 15 à $22 \mathrm{~mm}$, ou bien en forme de trapèze.

${ }^{114}$ Paris, Musée de l'Armée, nos. I 90 et I 91; Paris, Musée de l'Homme, no. 32.35 .10

11.5 Paris, Musée de l'Homme, no. 32.35.9.

${ }^{116}$ Paris, Musée de l'Armée, no. I 91; Vienne, Historisches Museum der Stadt Wien, no. 126.228 .

${ }^{117}$ Paris, Musée de l'Armée, no. I 87; Paris, Musée de l'Homme, no. 32.35.10.

$11:$ Paris, Musée de l'Homme, no. 32.35.9.

119 Paris, Musée de l'Armée, no. I 87.

${ }^{120}$ Paris, Musée de l'Homme, no. 32.35.9.

${ }^{121}$ Paris, Musée de l'Armée, no. I 91. 


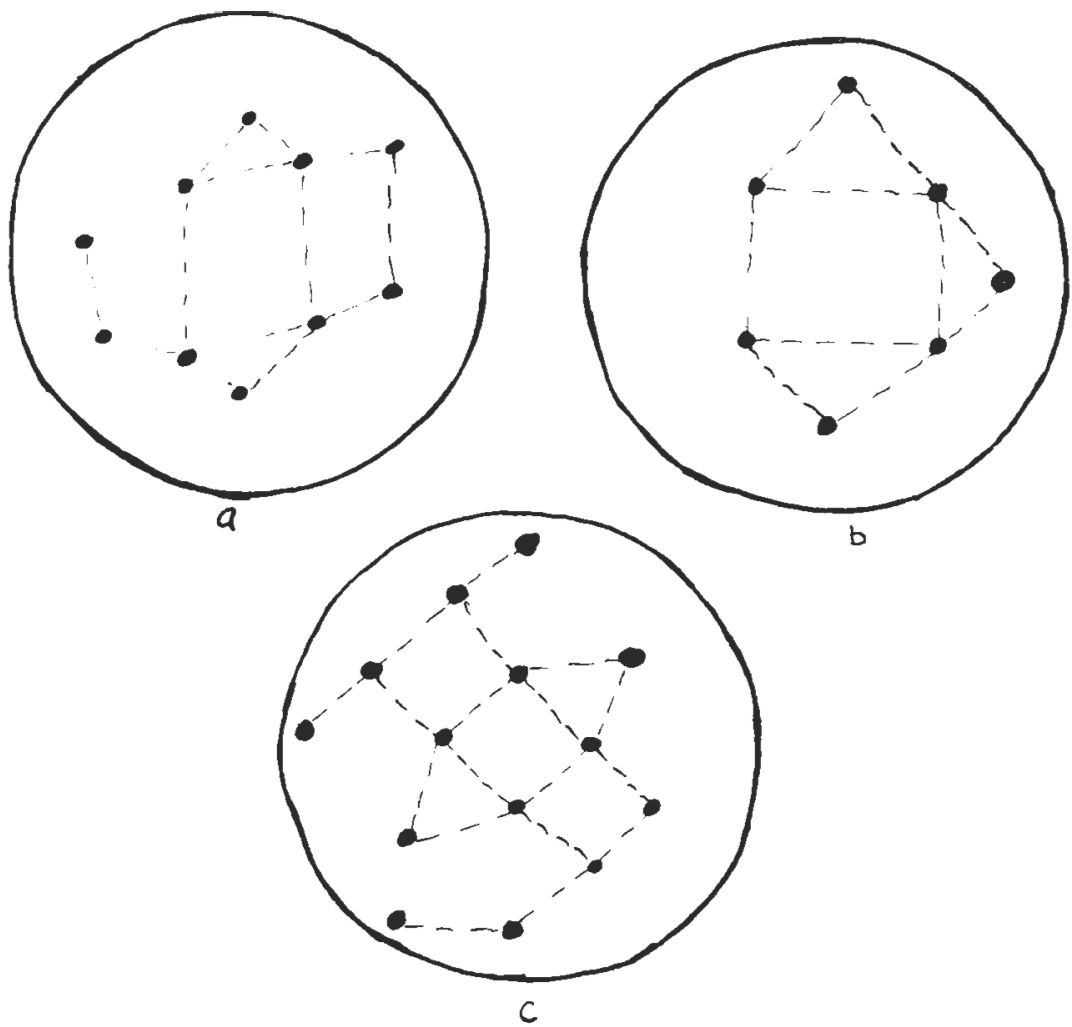

FIG. 21.-Exemples de la répartition des rivets sur les boucliers en osier tressé. ( $a=$ Paris, Musée de l'Armée, no. I 91; $b=$ Paris, Musée de l'Homme, no. 32.35.9; $c=$ Paris, Musée de l'Homme, no. 32.35.10.)

Cinq boucliers seulement possèdent encore des lanières ${ }^{12}$, sur les autres boucliers elles ont entièrement disparu. Elles sont en coton tressé, nouées aux anneaux, souvent décorées avec des glands. Un bouclier fait exception ${ }^{123}$, ses lanières sont en cuir tressé et c'est aussi par ce tressage qu'elles sont attachées aux anneaux.

Deux lanières sont toujours attachées aux quatre rivets centraux, leur longueur varie entre 200 et $250 \mathrm{~mm}$. Elles sont toujours placées en dia-

${ }^{122}$ Paris, Musée de l'Armée, nos. I 87, I 90 et I 91; Paris, Musée de l'Homme, no. 32.35.10; Vienne, Historisches Museum der Stadt Wien, no. 126.401.

${ }^{123}$ Vienne, Historisches Museum der Stadt Wien, no. 126.401. 
gonale (croisées), parfois tressées ensemble au point de leur croisement. Aux extrémités opposées de la surface interne peuvent être placées deux autres lanières, dont l'une peut avoir comme longueur entre 350 et $400 \mathrm{~mm}$, tandis que l'autre entre 180 et $260 \mathrm{~mm}$.

Éléments renforçant la solidité et l'efficacité du bouclier.-Il s'agit d'une bande en cuir ou en grosse étoffe qui couvre la tranche du bouclier et qui se replie, dépassant de $5 \mathrm{~mm}$ sur la surface externe et interne où elle est cousue sur la base. Sur certains boucliers ${ }^{124}$ c'est la matelassure en cuir qui est repliée sur la surface externe où elle dépasse aussi de $5 \mathrm{~mm}$ et couvre ainsi la tranche.

Eléments amortissant les chocs.-Sur les boucliers en osier tressé ce sont la matelassure et le coussinet. La matelassure est d'habitude en velours vert, violet ou rouge, sur un des boucliers observés ${ }^{125}$ elle est en toile et sur trois autres en cuir ${ }^{126}$. Elle couvre toute la surface interne où elle est cousue aux extrémités, par contre sur deux boucliers ${ }^{127}$ elle est repliée et dépasse de $5 \mathrm{~mm}$ sur la surface externe, protégeant ainsi la tranche de la base, comme nous l'avons déjà noté ci-dessus.

Le coussinet est en forme de carré dont les côtés varient entre 150 et $180 \mathrm{~mm}$, il est fait soit entièrement en velours, soit sa partie supérieure est en velours et sa partie inférieure est en toile ordinaire. Il est rempli de coton ce qui lui donne une épaisseur de cca $30 \mathrm{~mm}$. Sur ses angles, il est traversé par quatre rivets centraux qui le fixent ainsi, et entre les têtes des rivets et le coussinet est généralement placée une plaque, ayant une forme circulaire ou une forme décorative, comme nous l'avons déjà constaté ci-dessus.

\section{Boucliers en papier mâché}

Ce matériau est «obtenu en compressant dans un moule plusieurs feuilles de papier puis en les peignant et en les recouvrant d'un vernis» ${ }^{128}$.

${ }^{12+}$ Paris, Musée de l'Armée, no. I 89; Vienne, Historisches Museum der Stadt Wien, no. 126.401.

${ }^{125}$ Vienne, Historisches Museum der Stadt Wien, no. 126.402.

${ }^{126}$ Paris, Musée de l'Armée, no. I 89; Paris, Musée de I'Homme, no. 32.35.9; Vienne, Historisches Museum der Stadt Wien, no. 126.401.

${ }_{127}$ Paris, Musée de l'Armée, no. I 89; Vienne, Historisches Museum der Stadt Wien, no. 126.401 .

${ }_{128}$ Jeannine Auboyer: Les Arts de l'Inde et des pays indianisés, Paris, 1968, p. 153. Le vernis est fait «en Iran, d'une partie de résine de sandaque (sorte de thuya) et de trois parties d'huile de lin», voir Art appliqué islamique (texte pour 


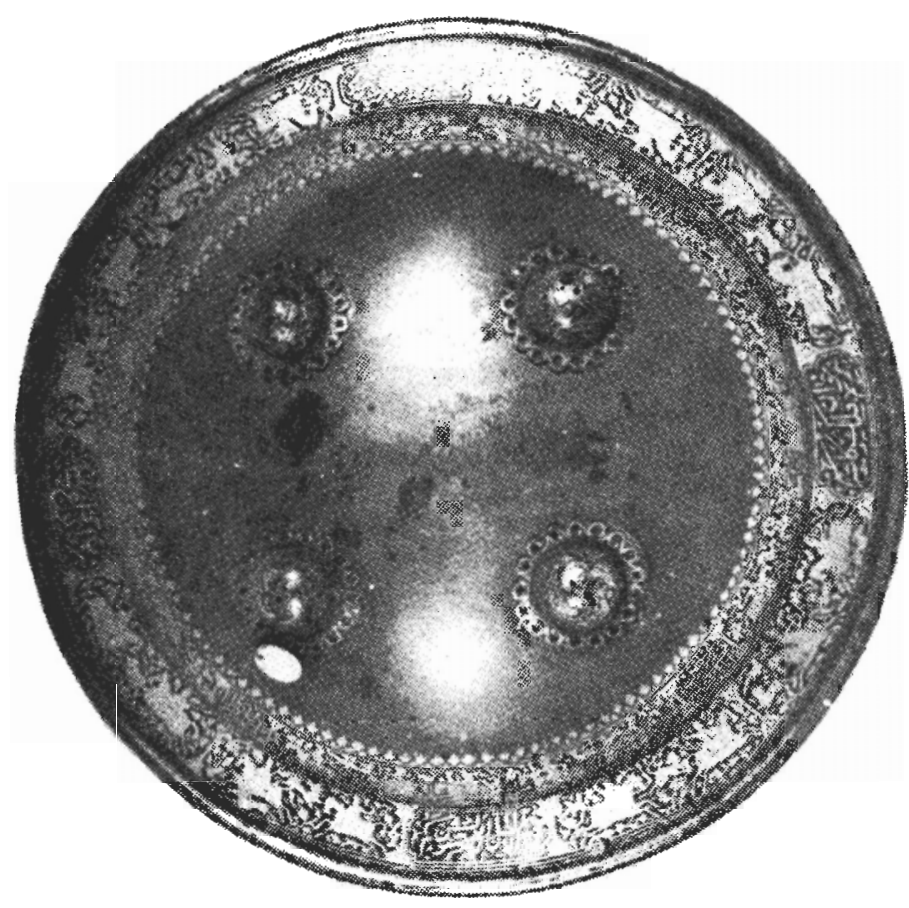

Frg. 22.-Bouclier en acier damassé. Iran, première moitié du XIX' siècle. Paris, Musée de l'Armée, no. 2852. (Phot. Service Photographique du Musée de l'Armée.)

Les boucliers en papier mâché n'étaient utilisés qu'à des fins décoratives, la matière n'étant pas très solide mais par contre très convenable pour être décorée. Leur production était, de plus, très bon marché ${ }^{129}$.

Nous n'avons trouvé pendant nos recherches qu'un seul bouclier de ce matériau ${ }^{130}$ et c'est la raison pour laquelle nous ne sommes pas en mesure de faire des observations d'ordre général. Néanmoins, à titre

une exposition au Musée d'Histoire de Berne, l'été 1971), p. 6. Au Pendjab le vernis est «obtained from Rhus Vernix, a shrub from which it exudes like gum», voir Rt. Hon. Lord Egerton of Thtтon: A Description of Indian and Oriental Armour, Londres, 1896, p. 68.

${ }^{129}$ Voir Thomas Holbein Hendey: Memorials of the Jeypore Exbibition, t. I, Londres, 1884, p. 2.

${ }^{130}$ Berne, Musée d'Histoire, no. 1285. 


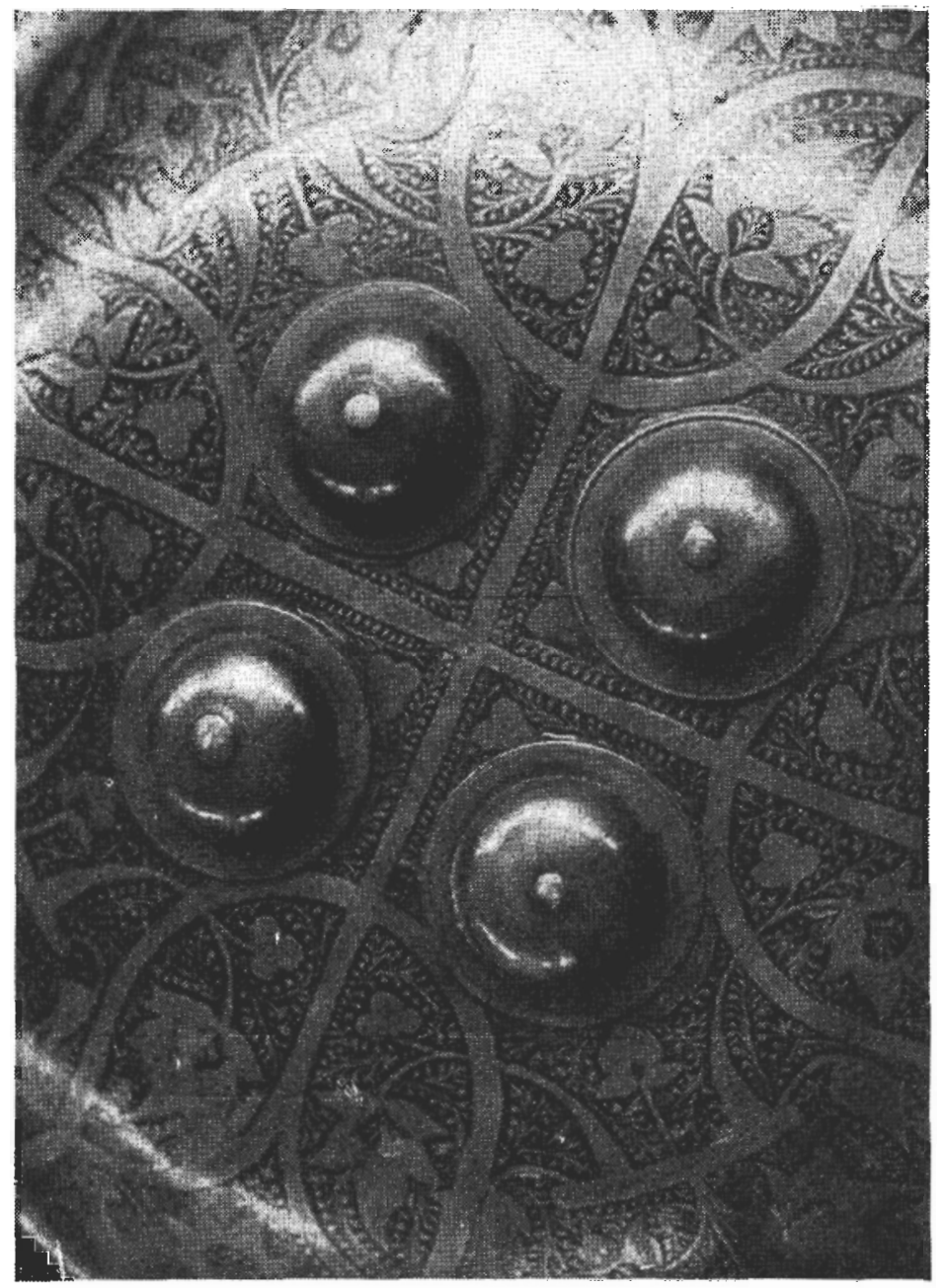

FIg. 23.-Détail d'un bouclier en laiton. Inde du Nord-Ouest, deuxième moitié du XIX' sic̀cle. Berne, Musée d'Histoire, no. 1370. (Phot. Kalus.) 
d'exemple, nous indiquons au moins ici les caractéristiques de cet exemplaire unique.

Sa base est profilée en forme de calotte, son diamètre est de $500 \mathrm{~mm}$, sa profondeur de $84 \mathrm{~mm}$ et son épaisseur est de $4 \mathrm{~mm}$.

On observe sur la surface externe quatre bossettes sculptées en forme de visage humain, leur diamètre est de $26 \mathrm{~mm}$ et leur hauteur de $13 \mathrm{~mm}$.

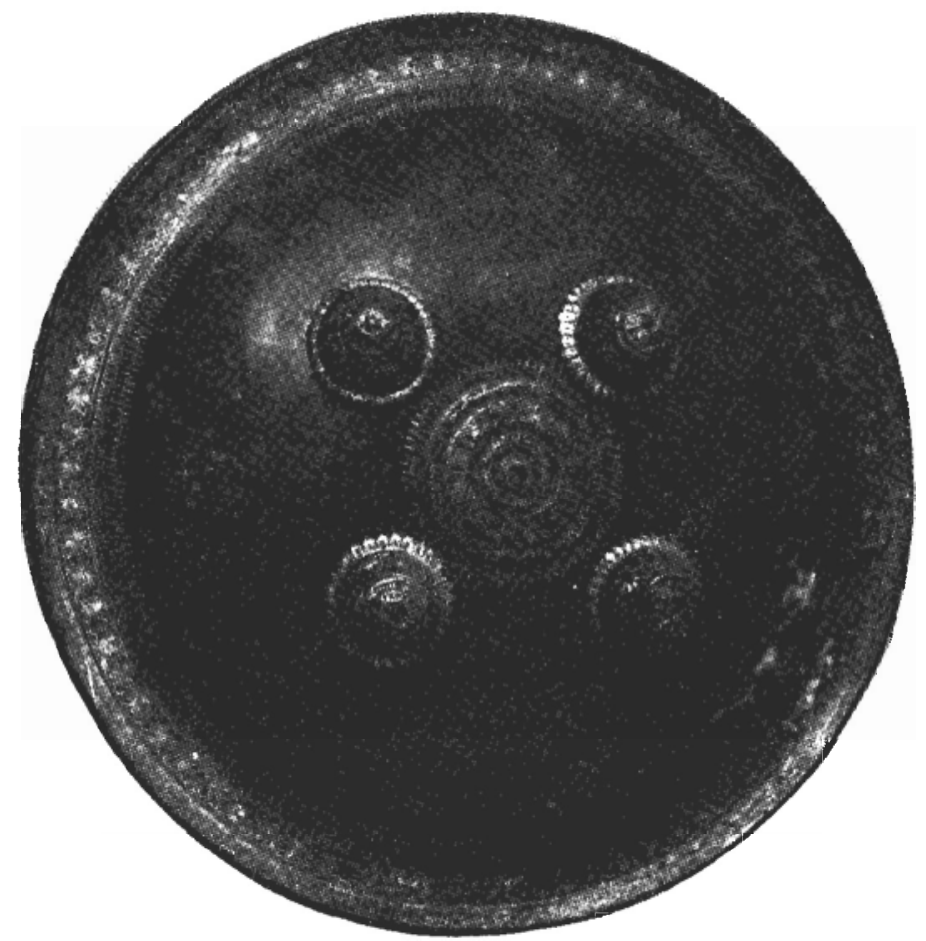

FIG. 24.-Bouclier en peau de rbinocéros translucide. Inde de l'Ouest, première moitié du XIX siècle. Berne, Musée d'Histoire, no. 967. (Phot. Kalus.)

Elles sont en cuivre jaune, elles sont rivetées et placées sur les angles d'un carré imaginaire dont les côtés sont de $115 \mathrm{~mm}$.

Sur la surface interne, les rivets des bossettes traversant la base s'élargissent en formant des têtes traversées par des anneaux qui sont très minces. A la place du coussinet est posé un carré de toile, traversé dans ses angles par des rivets qui le fixent. 


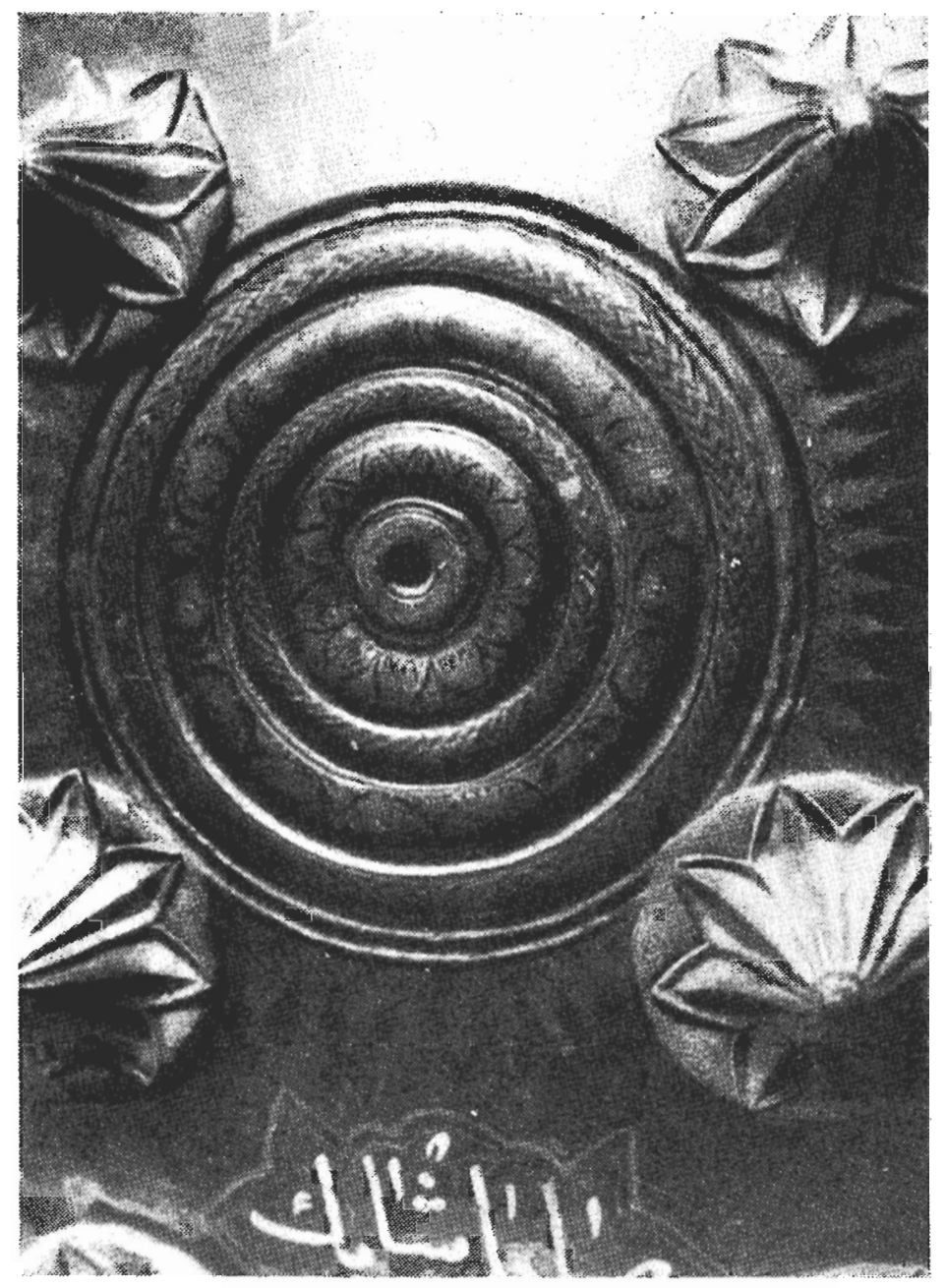

FIG. 25.-Détail du décor d'un bouclier en peau de rhinocéros translucide. Inde de l'Ouest (Sind?), première moitié du XIX siècle. Berne, Musée d'Histoire, no. 969. (Phot. Kalus.) 
Comparaison des éléments des boucliers en divers matériaux.-Dans cet partie, nous allons comparer les résultats de notre analyse morphologique dans le cadre des groupes déterminés par le matériau de la base, pouvant ainsi observer quels sont les éléments typiques pour tous les boucliers ou par contre seulement pour un ou quelques-uns de ces groupes ${ }^{131}$ (Figs. 23-26).

La base.-Le profil de la base des boucliers en métal et en peau prend presque toujours la forme d'une calotte sphérique, plus ou moins modifiée sur le bord ou sur le sommet. Les formes les plus répandues sont la calotte sphérique simple et la calotte sphérique légèrement aplatie sur le bord. Par contre, tandis que le bord des boucliers en métal est plutôt aplati, le bord de ceux en peau remonte généralement. Le bord d'un seul bouclier en métal remonte, lui aussi, mais il s'agit plutôt d'une imitation des boucliers en peau.

La peau est un matériau d'une grande flexibilité et par conséquent plus facile à profiler. C'est cela qui permet de faire remonter assez facilement le bord ce qui est nécessaire pour empêcher le glissement de l'arme de l'adversaire et ce qui rend la base du bouclier beaucoup plus résistante contre l'inflexion. Ce bord remonté est remplacé partiellement, sur les boucliers en métal, par une bordure rapportée.

Les diamètres des bases les plus fréquents sont pour les boucliers en osier entre 501 et $600 \mathrm{~mm}$, pour les boucliers en métal entre 401 et $500 \mathrm{~mm}$ et pour les boucliers en peau entre 401 et $450 \mathrm{~mm}$. Mais les boucliers en métal et en peau peuvent avoir un diamètre très petit, jusqu'à $255 \mathrm{~mm}$ pour ceux en métal et $260 \mathrm{~mm}$ pour ceux en peau, tandis que le diamètre le plus petit pour ceux en osier n'est que de $485 \mathrm{~mm}$. Le diamètre le plus grand de ces derniers atteint $620 \mathrm{~mm}$, de ceux en peau $580 \mathrm{~mm}$ et de ceux en métal $530 \mathrm{~mm}$.

Les profondeurs sont très diverses, les boucliers en peau peuvent être plus plats $(20 \mathrm{~mm})$ mais aussi plus bombés $(132 \mathrm{~mm})$ que ceux en métal ( 40 et $125 \mathrm{~mm}$ ). Pour les boucliers en métal la profondeur la plus fréquente est située entre 51 et $60 \mathrm{~mm}$ et entre 71 et $80 \mathrm{~mm}$, pour ceux en peau entre 61 et $70 \mathrm{~mm}$. Ici aussi, les boucliers en osier se distinguent un peu par leur profondeur qui est assez grande et qui varie entre 75 et $140 \mathrm{~mm}$.

L'épaisseur de la base des boucliers est donnée par le matériau utilisé, par le besoin de solidité ou par son épaisseur naturelle. Elle est pour le

${ }^{131}$ Nous ne tenons pas compte dans ces comparaisons des boucliers en papier mâché, à cause du manque d'un nombre suffisant d'objets qui nous permettrait des généralisations. 
métal de 1 à $3 \mathrm{~mm}$, pour la peau de 3 à $10 \mathrm{~mm}$ et pour l'osier de 5 à $8 \mathrm{~mm}$.

La légèreté des boucliers destinés au combat est un élément très important. Bien que ce fait ne pouvait pas être étudié par nous assez

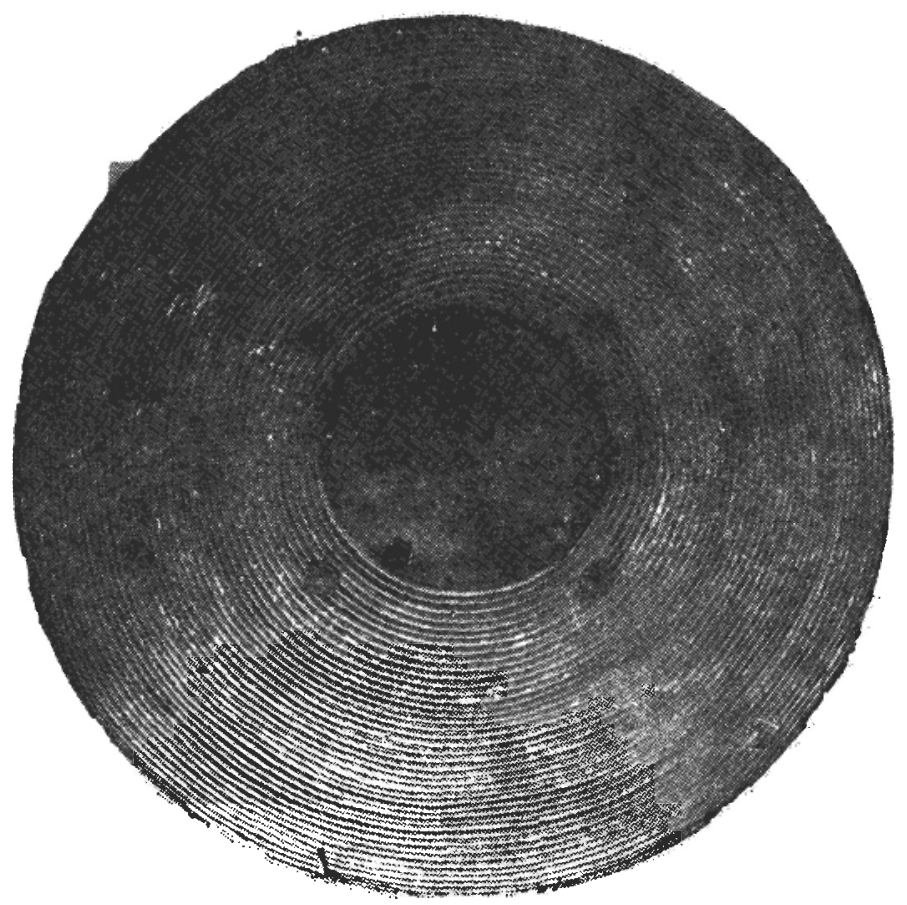

FIG, 26.-Bouclier en «osier» tressé. Iran ou Turquie, XVI" ou XVII ${ }^{c}$ siècle. Paris, Musée de l'Homme, no. 32.35.10. (Phot. Lemzaouda.)

systématiquement, il va de soi que le poids dépend du matériau employé. Les boucliers en métal sont les plus lourds et leur poids atteint $3470 \mathrm{~g}$, ce qui est un poids vraiment considérable. Par contre, il est surprenant qu'on ait trouvé un bouclier en peau qui pèse $2820 \mathrm{~g}$. Mais la fréquence du poids des boucliers en métal la plus courante se situe entre $1501 \mathrm{~g}$ et $2000 \mathrm{~g}$. Pour les boucliers en peau il peut être assez bas $(900 \mathrm{~g})$, mais nous avons aussi un bouclier en métal qui n'a que $980 \mathrm{~g}$. 
Parties rapportées.-Le système à bossettes est de loin le plus utilisé comme mécanisme permettant de porter et de manier les boucliers en peau et en métal: tous les boucliers en peau (sauf un qui n'a aucun mécanisme) et presque tous les boucliers en métal sont pourvus de ce système. Le système à petits rivets est typique pour les boucliers en osier tressé qui le possèdent tous, mais un bouclier en métal l'a également. Le type à poignée rivetée ou soudée est très rare et on ne le trouve que sur les boucliers en métal.

En général, on peut constater que les boucliers à six bossettes sont ceux dont le diamètre est assez grand. En ce qui concerne les boucliers en métal, ce ne sont pas ceux qui sont les plus larges (les deux boucliers à six bossettes ont des diamètres de 470 et $473 \mathrm{~mm}$ ), ceux en peau sont les plus larges mais peuvent aussi avoir un diamètre beaucoup plus petit (le plus petit est de $335 \mathrm{~mm}$ ). L'avantage du mécanisme à six bossettes sur celui à quatre bossettes est qu'il permet une prise du bouclier beaucoup plus solide et sûre.

La plupart des bossettes des boucliers en métal ont la forme d'un hémisphère et celles des boucliers en peau ont une forme très variable.

Les bossettes des boucliers en peau sont toujours fixées par des rivets, celles des boucliers en métal sont vissées sur la plupart des bouciers, mais on trouve aussi des bossettes qui sont rivetées.

Pour pouvoir être tenus, les boucliers ont des lanières. Elles sont en coton ou en cuir tressé pour les boucliers en osier, pour ceux en métal et en peau elles sont formées d'une bandelette en cuir, qui peut être aussi double ou triple et recouverte de velours.

Les lanières sur les boucliers en osier sont placées en diagonale, sur les boucliers en métal et en peau parallèlement, avec une exception pour les boucliers en métal et deux exceptions pour ceux en peau où elles sont placées en diagonale.

Les boucliers en osier ont deux lanières supplémentaires placées de chaque côté des lanières centrales. Les boucliers en peau peuvent avoir en plus des bretelles qui servent à les suspendre sur le dos ou sur l'épaule.

Une pointe saillante peut renforcer l'efficacité des boucliers en métal ou en osier en servant à freiner le glissement du coup d'une arme de taille et en permettant éventuellement d'utiliser le bouclier comme arme offensive.

Les boucliers en peau n'ont jamais de matelassure, la vraie matelassure ne se trouve que sur ceux en métal. Elle peut être remplacée par une «fausse» matelassure formée par une toile qui est collée sur la base. Cette «fausse» matelassure, en velours ou en cuir, est typique pour les boucliers en osier. 
Le coussinet des boucliers en osier est toujours carré, celui des boucliers en métal et en peau est carré ou rectangulaire. Les coussinets carrés des boucliers en peau ont leurs côtés presque toujours découpés, les coussinets rectangulaires peuvent avoir un des petits côtés décorés par un découpage décoratif.

Les parties rapportées peuvent être d'une solidité différente, suivant le but de l'utilisation du bouclier. Certaines manquent parfois soit parce qu'elles ont disparu, soit parce que le but de l'utilisation du bouclier ne les a pas exigées.

$$
* * *
$$

Les résultats que nous avons obtenus par l'analyse morphologique d'un certain nombre de boucliers circulaires de l'Orient musulman et que nous avons présentés dans cette étude ne sont que relatifs, tenant compte d'un nombre limité de boucliers que nous avons eu l'occasion d'étudier. Néanmoins, nous espérons avoir pu présenter une image assez fidèle du côté morphologique de ces boucliers, que des études ultérieures pourraient compléter ou éventuellement améliorer. 